\title{
EXPONENTIAL CONVERGENCE FOR SOME SPDES WITH LÉVY NOISES
}

\author{
YULIN SONG AND TIANGE XU
}

\begin{abstract}
In this paper, we generalize the Malliavin calculus for jump processes in the infinite-dimensional setting and obtain an integration by parts formula for jump processes on Hilbert spaces. By using this formula, we investigate derivative formula and exponential convergence for SPDEs driven by purely jump processes.
\end{abstract}

\section{Introduction and main results}

1.1. Introduction. Stochastic partial differential equations (SPDEs) driven by Lévy noises have been extensively studied in recent years; see [1], [2], [14], [17], [18], [19], [16], [21], [25] and references therein. For diffusions, the derivative formulas [7] (also called Bismut-Elwealthy-Li formula [13]) is a quite useful tool in various aspects such as functional inequalities [32], heat kernel estimates [7], strong Feller properties [10] and so on. Due to these various applications, many scholars have paid much attention to the analogous derivative formula for jump processes. For finite-dimensional jump processes, we refer to [3], [4], [20], [33], [35] etc. In most of these references, the Lévy measures of forced noises are always required to having absolutely continuous (parts) lower bounds w.r.t. the Lebesgue measure whose shift-invariance property plays an essential role. But in infinite-dimensional setting, there is no Lebesgue measure available. In [34], the authors investigated the strong Feller and coupling properties for linear SDEs driven by non-cylindrical Lévy processes on a Banach space equipped with a nice reference measure, which

Received September 22, 2016; received in final form January 20, 2017.

The first author is supported by NNSF (No.11501286), JSNSF (No. BK20150564) of China and Key Laboratory of Random Complex Structures and Data Science, Academy of Mathematics and Systems Science, Chinese Academy of Sciences (No. 2008DP173182).

2010 Mathematics Subject Classification. 60H07, 60H15. 
has quasi-invariance property. By Galerkin's approximation, a derivative formula was established in [28] for semilinear SPDEs forced by by non-cylindrical Lévy processes. However, the representation of the formula in [28] is cumbersome. So one aim of this work is to give a succinct formulation of derivative formula for a modified transitional probability function.

As an application of the derivative formula, we will study the long-time asymptotic behaviors of SPDEs with jumps (see Theorem 2 below). For this topic, in finite-dimensional case, the algebraic or exponential convergence of Lévy processes and SDEs forced by Lévy processes were studied in [8], [26], [27], [15], [22] and references therein. When the nonlinear terms of SPDEs forced by cylindrical $\alpha$-stable processes were bounded and Lipschitz continuous, exponential convergence were derived in [22], [24] and [23]. For stochastic Burgers equations, the exponential convergence was discussed in [12]. The exponential convergence for nonlinear SPDEs driven by non-cylindrical pure jump processes was also discussed in [28]. Another aim of this paper is to give the exponential convergence for SPDEs with Lévy noises.

1.2. Preliminaries. Let $(\mathbb{H},\langle\cdot, \cdot\rangle)$ be a separable Hilbert space and $\mu$ be a Gaussian measure on $\mathbb{H}$ with covariance operator $Q$, which is nonnegative, symmetric and with trace class. Its square root, denoted by $Q^{\frac{1}{2}}$, is a nonnegative and symmetric Hilbert-Schmidt operator. Let $\operatorname{Im} Q^{\frac{1}{2}}$ be the image space of $Q^{\frac{1}{2}}$, i.e., $\operatorname{Im} Q^{\frac{1}{2}}=\left\{Q^{\frac{1}{2}} x \mid x \in \mathbb{H}\right\}$. As is known, $\operatorname{Im} Q^{\frac{1}{2}}$ is a Hilbert space with the induced inner product

$$
\langle x, y\rangle_{0}:=\left\langle Q^{-\frac{1}{2}} x, Q^{-\frac{1}{2}} y\right\rangle, \quad x, y \in \operatorname{Im} Q^{\frac{1}{2}},
$$

where $Q^{-\frac{1}{2}}$ is the pseudo inverse of $Q$ in the case that it is not one-to-one, that is, for $h \in \operatorname{Im} Q^{\frac{1}{2}}$,

$$
Q^{-\frac{1}{2}} h=x, \quad \text { if } Q^{\frac{1}{2}} x=h \quad \text { and } \quad\|x\|=\inf \left\{\|y\|: Q^{\frac{1}{2}} y=h\right\} .
$$

As is known, the Gaussian measure $\mu$ has quasi-invariant property under the shift $z \mapsto z+h$ for any $h \in \operatorname{Im} Q^{\frac{1}{2}}$ (see Theorem 2.21 in [9]), that is, $\mu(\cdot+h)$ and $\mu$ are mutually absolutely continuous. The Randon-Nikodym derivative of $\mu(\cdot+h)$ w.r.t. $\mu$ is

$$
\varphi(z, h):=\frac{\mu(\mathrm{d} z+h)}{\mu(\mathrm{d} z)}=\exp \left\{\langle h, z\rangle_{0}-\frac{1}{2}\langle h, h\rangle_{0}\right\}, \quad \mu \text {-a.s. }
$$

Denote the eigenvectors of $Q$ by $\left\{e_{k}\right\}_{k \in \mathbb{N}}$, which can consist of an orthonormal basis of $\mathbb{H}$.

Let $\mathbb{W}$ be the space of all càdlàg functions from $[0, \infty)$ to $\mathbb{H}$ vanishing at 0 , which is endowed with the Skorohod topology. The $\sigma$-algebra $\mathcal{B}(\mathbb{W})$ is generated by all of the open sets of $\mathbb{W}$. Let $\mathbb{P}^{1}$ be a probability measure on $\mathbb{W}$ such that the coordinate process $L_{t}(w)=w(t)$ is a Lévy process with characteristic measure $\nu$. Let $\mathbb{P}^{2}$ be another probability measure on $\mathbb{W}$ such 
that the coordinate process $Z_{t}(w)=w_{t}$ is also a Lévy process with Lévy measure $\nu_{Z}$ satisfying $\int_{\mathbb{H}}|z|^{2} \nu_{Z}(\mathrm{~d} z)<\infty$. Throughout this paper, for Lévy measure $\nu$, we assume that

$\left(\mathbf{H}_{\nu}\right)$ There exists a Fréchet differentiable function $\rho: \mathbb{H} \rightarrow(0, \infty)$ with bounded derivative such that

$$
\nu(\mathrm{d} z)=\rho(z) \mu(\mathrm{d} z), \quad \lambda:=\nu(\mathbb{H}) \in(0,+\infty), \quad \text { and } \quad \int_{\mathbb{H}}|z|^{2} \nu(\mathrm{d} z)<\infty .
$$

Define a probability space as

$$
(\Omega, \mathcal{F}, \mathbb{P}):=\left(\mathbb{W} \times \mathbb{W}, \mathcal{B}(\mathbb{W}) \times \mathcal{B}(\mathbb{W}), \mathbb{P}^{1} \times \mathbb{P}^{2}\right)
$$

Let $\left\{\mathcal{F}_{t}\right\}_{t \geq 0}$ be the filtration generated by $L$ and $Z$. Denote the jump measure of $L$ by $N(\mathrm{~d} z, \mathrm{~d} t) . \tilde{N}(\mathrm{~d} z, \mathrm{~d} t):=N(\mathrm{~d} z, \mathrm{~d} t)-\nu(\mathrm{d} z) \mathrm{d} t$ is the martingale measure. Let $N_{t}:=N([0, t] \times \mathbb{H})$ stand for the associated counting process.

In this paper, we consider the following stochastic equation on $\mathbb{H}$

$$
\left\{\begin{array}{l}
\mathrm{d} X_{t}=A X_{t} \mathrm{~d} t+F\left(X_{t}\right) \mathrm{d} t+\mathrm{d} L_{t}+\mathrm{d} Z_{t}, \\
X_{0}=x,
\end{array}\right.
$$

where $A: \mathcal{D}(A) \subset \mathbb{H} \rightarrow \mathbb{H}$ is an adjoint, unbounded and linear operator generating a $C_{0}$-semigroup $\left\{S_{t}\right\}_{t \geq 0}$ and $F: \mathbb{H} \rightarrow \mathbb{H}$ is bounded and Lipschitz continuous. Then the mild solution of Eq. (2) exists uniquely (see [21, Theorem 9.7]) and can be formulated as

$$
\begin{aligned}
X_{t}^{x}= & S(t) x+\int_{0}^{t} S(t-s) F\left(X_{s}^{x}\right) \mathrm{d} s+\int_{0}^{t} S(t-s) \mathrm{d} L_{s} \\
& +\int_{0}^{t} S(t-s) \mathrm{d} Z_{s} .
\end{aligned}
$$

Let $\left\{P_{t}(x, \cdot)\right\}_{t \geq 0}$ and $\left\{P_{t}\right\}_{t \geq 0}$ be the transition probability measures and transition semigroups respectively.

Now we give some notations for later use. Let $\mathcal{B}(\mathbb{H})$ be the $\sigma$-algebra generated by all of the open subsets of $\mathbb{H}$. For $i=1,2$, we employ $C_{b}^{i}(\mathbb{H}, \mathbb{H})\left(C_{b}^{i}(\mathbb{H})\right)$ to denote the family of $\mathbb{H}$-valued (real-valued) $i$ th Fréchet differentiable functions $f$ such that $f$ and its derivatives are bounded and continuous. Let $\mathcal{B}_{b}(\mathbb{H})$ be the Banach space of bounded Borel-measurable functions $f: \mathbb{H} \rightarrow \mathbb{R}$ with the supremum norm $\|f\|_{\infty}:=\sup _{y \in \mathbb{H}}|f(y)|$. Let $\mathcal{P}(\mathbb{H})$ be the set of probabilities on $(\mathbb{H}, \mathcal{B}(\mathbb{H}))$. Recall that the total variation distance between two finite measures $\mu_{1}, \mu_{2}$ is defined by

$$
\left\|\mu_{1}-\mu_{2}\right\|_{\text {Var }}:=\frac{1}{2} \sup _{f \in \mathcal{B}_{b}(\mathbb{H}),\|f\|_{\infty} \leq 1}\left|\mu_{1}(f)-\mu_{2}(f)\right| .
$$

The norm of a linear bounded operator $P: \mathbb{H} \rightarrow \mathbb{H}$ is defined by

$$
\|P\|:=\sup _{y \in \mathbb{H},|y|=1}|P y| \text {. }
$$


We define the operators $\left\{P_{t}^{1}\right\}_{t \geq 0}$ which were first introduced in [31] as

$$
P_{t}^{1} f(x):=\mathbb{E}\left\{f\left(X_{t}^{x}\right) I_{\left[N_{t} \geq 1\right]}\right\}, \quad x \in \mathbb{H}, t \geq 0, f \in \mathcal{B}_{b}(\mathbb{H}),
$$

where $I_{\left[N_{t} \geq 1\right]}$ is an indicator function.

1.3. Main results. We list the hypotheses for Equ. (2):

- $\left(\mathbf{H}_{A}\right) A$ is a dissipative operator defined by

$$
A=\sum_{k \geq 1}\left(-\gamma_{k}\right) e_{k} \otimes e_{k},
$$

for $0<\gamma_{1} \leq \gamma_{2} \leq \cdots \leq \gamma_{k} \leq \cdots$ and $\gamma_{k} \rightarrow \infty$ as $k \rightarrow \infty$.

- $\left(\mathbf{H}_{S Q}\right) \operatorname{Im} S(t) \subset \operatorname{Im} Q$ and $\int_{0}^{t}\left\|Q^{-1} S(s)\right\| \mathrm{d} s<\infty$ hold for any $t>0$.

We have the following main results.

TheOREM 1. Let $F \in C_{b}^{1}(\mathbb{H}, \mathbb{H})$ with $\nabla F$ Lipschitz continuous. Assume $\left(\mathbf{H}_{\nu}\right),\left(\mathbf{H}_{A}\right)$ and $\left(\mathbf{H}_{S Q}\right)$ hold. Then for $f \in C_{b}^{2}(\mathbb{H})$ and $\xi \in \mathbb{H}$,

$$
\begin{aligned}
\nabla_{\xi} P_{t}^{1} f(x)= & -\mathbb{E}\left\{f\left(X_{t}^{x}\right) \frac{I_{\left[N_{t} \geq 1\right]}}{N_{t}}\right. \\
& \left.\times \int_{0}^{t} \int_{\mathbb{H}}\left(\left\langle z, Q^{-1} J_{s} \xi\right\rangle+\left\langle\nabla \log \rho(z), J_{s} \xi\right\rangle\right) \tilde{N}(\mathrm{~d} z, \mathrm{~d} s)\right\},
\end{aligned}
$$

where $J_{t} \xi$ is the derivative of $X_{t}^{x}$ w.r.t. the initial value $x$ along the direction $\xi$.

REMARK 1. Compared with Theorem 1.2 in [28], this modified formula is much succinct. Meanwhile, the technical conditions of the Lévy measure $\nu$ are also relaxed. The price we pay here is the loss of strong Feller property of $P_{t}$. Fortunately, this formula can be applied to prove the following exponential convergence of $P_{t}$.

THEOREM 2. Let $F$ be a Lipschitz continuous function with Lipschitz constant $\|F\|_{\text {Lip. Assume }}\left(\mathbf{H}_{\nu}\right),\left(\mathbf{H}_{A}\right)$ and $\left(\mathbf{H}_{S Q}\right)$ hold. If $\gamma_{1}>\|F\|_{\text {Lip }}$ and $\lim _{t \rightarrow \infty} \frac{\int_{0}^{t}\left\|Q^{-1} S(s)\right\|^{2} \mathrm{~d} s}{t}<\infty$, then there exists a constant $C>0$ such that for $x$ and $y \in \mathbb{H}$,

$$
\left\|P_{t}(x, \cdot)-P_{t}(y, \cdot)\right\|_{\text {Var }} \leq C(1+|x-y|) \exp \left\{-\frac{\lambda\left(\gamma_{1}-\|F\|_{\text {Lip }}\right)}{\lambda+\gamma_{1}-\|F\|_{\text {Lip }}} t\right\} .
$$

REMARK 2. According to Theorem 16.2 in [21], there is a unique invariant measure $\Xi$ for (3). Integrating both sides of (7) w.r.t. $\Xi(\mathrm{d} y)$, we immediately have

$$
\left\|P_{t}(x, \cdot)-\Xi\right\|_{\text {Var }} \leq C(1+|x|) \exp \left\{-\frac{\lambda\left(\gamma_{1}-\|F\|_{\text {Lip }}\right)}{\lambda+\gamma_{1}-\|F\|_{\text {Lip }}} t\right\}, \quad \forall x \in \mathbb{H} .
$$

We should point out that the exponential convergence (8) is not the same with the one discussed in [21, Theorem 16.2], since here we use the total variation norm not the so-called Fortet-Mourier norm (see [21, Definition 16.2]). 
EXAMPLE 1. Consider the following stochastic semilinear equation on $D=$ $[0, T]^{d}$ with $d \geq 1$ and the Dirichlet boundary condition:

$$
\left\{\begin{array}{l}
\mathrm{d} X(t, \xi)=[\Delta X(t, \xi)+F(X(t, \xi))] \mathrm{d} t+\mathrm{d} Z_{t}(\xi), \\
X(0, \xi)=x(\xi) \\
X(t, \xi)=0, \quad \xi \in \partial D
\end{array}\right.
$$

where $\left\{Z_{t}\right\}$ is a square integrable Lévy processes valued on $\mathbb{H}:=L^{2}(D)$ and $F: \mathbb{H} \rightarrow \mathbb{H}$ is Lipschitz continuous. It is clear that $\Delta$ with a Dirichlet boundary condition has the following eigenfunctions

$$
e_{k}(\xi)=\left(\frac{2}{\pi}\right)^{\frac{d}{2}} \sin \left(k_{1} \xi_{1}\right) \sin \left(k_{2} \xi_{2}\right) \cdots \sin \left(k_{d} \xi_{d}\right), \quad k \in \mathbb{N}^{d}, \xi \in D .
$$

It is known that $\Delta e_{k}=-|k|^{2} e_{k}$, i.e.

$$
\gamma_{k}=|k|^{2}=k_{1}^{2}+k_{2}^{2}+\cdots+k_{d}^{2}, \quad \text { for all } k \in \mathbb{N}^{d} .
$$

We study the dynamics defined by $(9)$ in the space $\mathbb{H}=L^{2}(D)$ with orthonormal basis $\left\{e_{k}\right\}_{k \in \mathbb{N}^{d}}$. For $0<\delta<\frac{1}{2}$, the fractional power $(-\Delta)^{\delta}$ of $-\Delta$ is defined by

$$
(-\Delta)^{\delta}=\frac{1}{\Gamma(\delta)} \int_{0}^{\infty} t^{-\delta} S(t) \mathrm{d} t,
$$

where $\Gamma$ is the Euler function. Due to Proposition A.12 in [9], we have $S(t) \mathbb{H} \subset$ $\mathcal{D}\left((-\Delta)^{\delta}\right)$ and for any $t>0$,

$$
\left\|(-\Delta)^{\delta} S(t)\right\| \leq C_{\delta} t^{-\delta}
$$

for a suitable positive constant $C_{\delta}$. If the operator $Q$ is defined as $Q:=$ $\left((-\Delta)^{\delta}\right)^{-1}$, then we have $S(t) \mathbb{H} \subset \operatorname{Im} Q$. Moreover,

$$
\lim _{t \rightarrow \infty} \frac{\int_{0}^{t}\left\|Q^{-1} S(s)\right\|^{2} \mathrm{~d} s}{t} \leq \lim _{t \rightarrow \infty} \frac{C_{\delta}^{2} \int_{0}^{t} s^{-2 \delta} \mathrm{d} s}{t}=\lim _{t \rightarrow \infty} \frac{C_{\delta}^{2} t^{1-2 \delta}}{t}=0 .
$$

The rest of this paper is organized as follows: in Section 2, we shall investigate an integration by parts formula for jump processes in infinite-dimensional case; the proofs of the main results will be presented in Section 3.

\section{Integration by parts formula}

The Malliavin calculus has played an important role in many fields as one of powerful tools in infinite-dimensional analysis. An integration by parts formula, which plays an important role in Malliavin calculus, can be used to derive the derivative formula. In finite-dimensional case, the integration by part formulas for jump processes was studied in [6], [5], [4], [20], [30], [29] and so on. But so far, there are few references studying the formula for pure jump processes in infinite-dimensional case. 
2.1. Girsanov's theorem. Denote

$$
\begin{aligned}
\mathbb{V}_{0}= & \{V: \Omega \times[0, T] \rightarrow \operatorname{Im} Q \mid \\
& \left.V \text { is predictable and } \sup _{\omega \in \Omega} \sup _{t \leq T}\left|Q^{-1} V(\omega, t)\right|<\infty\right\},
\end{aligned}
$$

and

$$
\begin{aligned}
\mathbb{V}= & \{V: \Omega \times[0, T] \rightarrow \operatorname{Im} Q \mid \\
& \left.V \text { is predictable and } \int_{0}^{T} \mathbb{E}\left(\left|Q^{-1} V(\cdot, t)\right|+|V(\cdot, t)|^{2}\right) \mathrm{d} t<\infty\right\} .
\end{aligned}
$$

In the following, we will drop " $\omega$ " in $V$ for the sake of writing. For any $V \in \mathbb{V}$, define a perturbed random measure $N^{\varepsilon}$ by

$$
N^{\varepsilon}(\Gamma \times[0, t])=\int_{0}^{t} \int_{\mathbb{H}} I_{\Gamma}(z+\varepsilon V(s)) N(\mathrm{~d} z, \mathrm{~d} s), \quad \Gamma \in \mathcal{B}(\mathbb{H}) .
$$

For $\varepsilon>0$, let

$$
\lambda^{\varepsilon}(t, z):=\varphi(z, \varepsilon V(t)) \frac{\rho(z+\varepsilon V(t))}{\rho(z)}, \quad \forall z \in \mathbb{H}, \forall t \in[0, T],
$$

where $\varphi$ is defined by (1). Let

$$
\Theta_{t}^{\varepsilon}:=\exp \left\{\int_{0}^{t} \int_{\mathbb{H}} \log \lambda^{\varepsilon}(s, z) N(\mathrm{~d} z, \mathrm{~d} s)-\int_{0}^{t} \int_{\mathbb{H}}\left(\lambda^{\varepsilon}(s, z)-1\right) \nu(\mathrm{d} z) \mathrm{d} s\right\} .
$$

Recalling that $\nu$ is a finite measure, by Itô's formula we can easily check that $\left\{\Theta_{t}^{\varepsilon}, \mathcal{F}_{t}\right\}_{t \leq T}$ is a uniformly integrative martingale. Moreover, $\Theta_{t}^{\varepsilon}$ can also be written as

$$
\Theta_{t}^{\varepsilon}=1+\int_{0}^{t} \int_{\mathbb{H}} \Theta_{s-}^{\varepsilon}\left(\lambda^{\varepsilon}(s, z)-1\right) \tilde{N}(\mathrm{~d} z, \mathrm{~d} s) .
$$

By Girsanov's theorem (see [11, Theorem 12.21]), there exists a probability measure $\mathbb{P}^{\varepsilon}$ such that

$$
\left.\frac{\mathrm{d} \mathbb{P}^{\varepsilon}}{\mathrm{d} \mathbb{P}}\right|_{\mathcal{F}_{t}}=\Theta_{t}^{\varepsilon}, \quad t \leq T .
$$

Then we have the following results.

LEMMA 3. Let $\left(\mathbf{H}_{\nu}\right)$ hold. Then for any $V \in \mathbb{V}, \nu$ is the characteristic measure of $N^{\varepsilon}(\mathrm{d} z, \mathrm{~d} t)$ under $\mathbb{P}^{\varepsilon}$.

Proof. For any bounded test function $\phi:[0, T] \times \mathbb{H} \rightarrow \mathbb{R}$, define

$$
Y_{t}^{\varepsilon}:=\exp \left\{\int_{0}^{t} \int_{\mathbb{H}} \phi(s, z) N^{\varepsilon}(\mathrm{d} z, \mathrm{~d} s)\right\}, \quad G_{t}^{\varepsilon}:=Y_{t}^{\varepsilon} \Theta_{t}^{\varepsilon} .
$$


By Itô's formula, we have

$$
\begin{aligned}
Y_{t}^{\varepsilon} & =\exp \left\{\int_{0}^{t} \int_{\mathbb{H}} \phi(s, z) N^{\varepsilon}(\mathrm{d} z, \mathrm{~d} s)\right\} \\
& =\exp \left\{\int_{0}^{t} \int_{\mathbb{H}} \phi(s, z+\varepsilon V(s)) N(\mathrm{~d} z, \mathrm{~d} s)\right\} \\
& =1+\int_{0}^{t} \int_{\mathbb{H}} Y_{s-}^{\varepsilon}\left(e^{\phi(s, z+\varepsilon V(s))}-1\right) N(\mathrm{~d} z, \mathrm{~d} s),
\end{aligned}
$$

and

$$
\left[Y^{\varepsilon}, \Theta^{\varepsilon}\right]_{t}=\int_{0}^{t} \int_{\mathbb{H}} Y_{s-}^{\varepsilon} \Theta_{s-}^{\varepsilon}\left(e^{\phi(s, z+\varepsilon V(s))}-1\right)\left(\lambda^{\varepsilon}(s, z)-1\right) N(\mathrm{~d} z, \mathrm{~d} s) .
$$

It follows from (16), (17) and Itô's formula that

$$
\begin{aligned}
G_{t}^{\varepsilon}=1 & +\int_{0}^{t} \int_{\mathbb{H}} Y_{s-}^{\varepsilon} \mathrm{d} \Theta_{s}^{\varepsilon}+\int_{0}^{t} \int_{\mathbb{H}} \Theta_{s-}^{\varepsilon} \mathrm{d} Y_{s}^{\varepsilon}+\left[Y^{\varepsilon}, \Theta^{\varepsilon}\right]_{t} \\
= & +\int_{0}^{t} \int_{\mathbb{H}} Y_{s-}^{\varepsilon} \mathrm{d} \Theta_{s}^{\varepsilon}+\int_{0}^{t} \int_{\mathbb{H}} \Theta_{s-}^{\varepsilon} Y_{s-}^{\varepsilon}\left(e^{\phi(s, z+\varepsilon V(s))}-1\right) N(\mathrm{~d} z, \mathrm{~d} s) \\
& +\int_{0}^{t} \int_{\mathbb{H}} Y_{s-}^{\varepsilon} \Theta_{s-}^{\varepsilon}\left(e^{\phi(s, z+\varepsilon V(s))}-1\right)\left(\lambda^{\varepsilon}(s, z)-1\right) N(\mathrm{~d} z, \mathrm{~d} s) \\
= & 1+\int_{0}^{t} Y_{s-}^{\varepsilon} \mathrm{d} \Theta_{s}^{\varepsilon}+\int_{0}^{t} \int_{\mathbb{H}} G_{s-}^{\varepsilon}\left(e^{\phi(s, z+\varepsilon V(s))}-1\right) \lambda^{\varepsilon}(s, z) N(\mathrm{~d} z, \mathrm{~d} s) .
\end{aligned}
$$

By (13) and (11), we arrive at

$$
\begin{aligned}
\mathbb{E} G_{t}^{\varepsilon} & =1+\mathbb{E} \int_{0}^{t} \int_{\mathbb{H}} G_{s-}^{\varepsilon}\left(e^{\phi(s, z+\varepsilon V(s))}-1\right) \lambda^{\varepsilon}(s, z) N(\mathrm{~d} z, \mathrm{~d} s) \\
& =1+\mathbb{E} \int_{0}^{t} \int_{\mathbb{H}} G_{s}^{\varepsilon}\left(e^{\phi(s, z+\varepsilon V(s))}-1\right) \varphi(z, \varepsilon V(s)) \frac{\rho(z+\varepsilon V(s))}{\rho(z)} \rho(z) \mu(\mathrm{d} z) \mathrm{d} s \\
& =1+\mathbb{E} \int_{0}^{t} \int_{\mathbb{H}} G_{s}^{\varepsilon}\left(e^{\phi(s, z+\varepsilon V(s))}-1\right) \varphi(z, \varepsilon V(s)) \rho(z+\varepsilon V(s)) \mu(\mathrm{d} z) \mathrm{d} s \\
& =1+\mathbb{E} \int_{0}^{t} \int_{\mathbb{H}} G_{s}^{\varepsilon}\left(e^{\phi(s, z+\varepsilon V(s))}-1\right) \rho(z+\varepsilon V(s)) \mu(\mathrm{d} z+\varepsilon V(s)) \mathrm{d} s \\
& =1+\int_{0}^{t} \int_{\mathbb{H}}\left(e^{\phi(s, z)}-1\right) \rho(z) \mu(\mathrm{d} z) \mathbb{E} G_{s}^{\varepsilon} \mathrm{d} s .
\end{aligned}
$$

Therefore,

$$
\mathbb{E} G_{t}^{\varepsilon}=\exp \left\{\int_{0}^{t} \int_{\mathbb{H}}\left(e^{\phi(s, z)}-1\right) \nu(\mathrm{d} z) \mathrm{d} s\right\}
$$


Combining this with (14) and (15), we have

$$
\begin{aligned}
\mathbb{E}^{\varepsilon} & \exp \left\{\int_{0}^{t} \int_{\mathbb{H}} \phi(s, z) N^{\varepsilon}(\mathrm{d} z, \mathrm{~d} s)\right\} \\
= & \exp \left\{\int_{0}^{t} \int_{\mathbb{H}}\left(e^{\phi(s, z)}-1\right) \nu(\mathrm{d} z) \mathrm{d} s\right\} .
\end{aligned}
$$

That is, $\nu(\mathrm{d} z)$ is the characteristic measure of $N^{\varepsilon}(\mathrm{d} z, \mathrm{~d} t)$ under $\mathbb{P}^{\varepsilon}$.

LEMma 4. Let $\left(\mathbf{H}_{\nu}\right)$ hold. Assume there exists a constant $\delta>0$ such that $\rho(z) \geq \delta$ for $\forall z \in \mathbb{H}$. Then for any $V \in \mathbb{V}_{0}$,

$$
\sup _{\varepsilon<1} \mathbb{E}\left\{\sup _{0 \leq t \leq T}\left|\frac{\Theta_{t}^{\varepsilon}-1}{\varepsilon}\right|^{2}\right\}<\infty .
$$

Proof. Since $V \in \mathbb{V}_{0}$, then one has

$$
\sup _{\omega \in \Omega, t \in[0, T]}\left(|V(\omega, t)|+\left|Q^{-1} V(\omega, t)\right|\right) \leq C(T),
$$

where $C(T)$, independent of $\varepsilon$, is a generic constant whose values might change from line to line. For any $t \in[0, T], z \in \mathbb{H}$ and $\varepsilon \in(0,1)$, it follows from (1) and (18) that

$$
\begin{aligned}
\varphi^{2}(z, \varepsilon V(t)) & =\varphi(z, 2 \varepsilon V(t)) \exp \left\{\varepsilon^{2}\left\langle V(t), Q^{-1} V(t)\right\rangle\right\} \\
& \leq C(T) \varphi(z, 2 \varepsilon V(t)) .
\end{aligned}
$$

Then by mean value theorem, we have

$$
\begin{aligned}
I_{1} & :=\int_{\mathbb{H}} \frac{\varphi^{2}(z, \varepsilon V(t))}{\rho(z)}\left|\frac{\rho(z+\varepsilon V(t))-\rho(z)}{\varepsilon}\right|^{2} \mu(\mathrm{d} z) \\
& \leq \frac{1}{\delta}\|\nabla \rho\|_{\infty}^{2}|V(t)|^{2} \int_{\mathbb{H}} \varphi^{2}(z, \varepsilon V(t)) \mu(\mathrm{d} z) \\
& \leq \frac{C(T)}{\delta}\|\nabla \rho\|_{\infty}^{2} \int_{\mathbb{H}} \varphi(z, 2 \varepsilon V(t)) \mu(\mathrm{d} z) \\
& \leq C(T) .
\end{aligned}
$$

Also, there exists a constant $\varepsilon_{1} \in(0, \varepsilon)$ such that

$$
\begin{aligned}
I_{2}:= & \int_{\mathbb{H}}\left|\frac{\varphi(z, \varepsilon V(t))-1}{\varepsilon}\right|^{2} \rho(z) \mu(\mathrm{d} z) \\
= & \int_{\mathbb{H}} \varphi^{2}\left(z, \varepsilon_{1} V(t)\right)\left|\left\langle z-\varepsilon_{1} V(s), Q^{-1} V(t)\right\rangle\right|^{2} \rho(z) \mu(\mathrm{d} z) \\
\leq & C(T) \int_{\mathbb{H}} \varphi^{2}\left(z, \varepsilon_{1} V(t)\right)|z|^{2} \rho(z) \mu(\mathrm{d} z) \\
& +C(T) \int_{\mathbb{H}} \varphi^{2}\left(z, \varepsilon_{1} V(t)\right) \rho(z) \mu(\mathrm{d} z)
\end{aligned}
$$




$$
\begin{aligned}
\leq & C(T) \int_{\mathbb{H}} \varphi\left(z, 2 \varepsilon_{1} V(t)\right)|z|^{2} \rho(z) \mu(\mathrm{d} z) \\
& +C(T) \int_{\mathbb{H}} \varphi\left(z, 2 \varepsilon_{1} V(t)\right) \rho(z) \mu(\mathrm{d} z) .
\end{aligned}
$$

By triangle inequality and mean value theorem, one has

$$
\begin{aligned}
I_{3}:= & \int_{\mathbb{H}} \varphi\left(z, 2 \varepsilon_{1} V(t)\right)|z|^{2} \rho(z) \mu(\mathrm{d} z) \\
= & \int_{\mathbb{H}}|z|^{2} \rho(z) \mu\left(\mathrm{d} z+2 \varepsilon_{1} V(t)\right) \\
\leq & 2 \int_{\mathbb{H}}\left|z+2 \varepsilon_{1} V(t)\right|^{2}\left|\rho\left(z+2 \varepsilon_{1} V(t)\right)-\rho(z)\right| \mu\left(\mathrm{d} z+2 \varepsilon_{1} V(t)\right) \\
& +2 \int_{\mathbb{H}}\left|z+2 \varepsilon_{1} V(t)\right|^{2} \rho\left(z+2 \varepsilon_{1} V(t)\right) \mu\left(\mathrm{d} z+2 \varepsilon_{1} V(t)\right) \\
& +2 \int_{\mathbb{H}}\left|2 \varepsilon_{1} V(t)\right|^{2}\left|\rho\left(z+2 \varepsilon_{1} V(t)\right)-\rho(z)\right| \mu\left(\mathrm{d} z+2 \varepsilon_{1} V(t)\right) \\
& +2 \int_{\mathbb{H}}\left|2 \varepsilon_{1} V(t)\right|^{2} \rho\left(z+2 \varepsilon_{1} V(t)\right) \mu\left(\mathrm{d} z+2 \varepsilon_{1} V(t)\right) \\
\leq & C(T)\|\nabla \rho\|_{\infty} \int_{\mathbb{H}}|z|^{2} \mu(\mathrm{d} z)+2 \int_{\mathbb{H}}|z|^{2} \rho(z) \mu(\mathrm{d} z) \\
& +C(T)\|\nabla \rho\|_{\infty}+C(T) \int_{\mathbb{H}} \rho(z) \mu(\mathrm{d} z) \\
\leq & C(T) .
\end{aligned}
$$

Similar arguments give that

$$
I_{4}:=\int_{\mathbb{H}} \varphi\left(z, 2 \varepsilon_{1} V(t)\right) \rho(z) \mu(\mathrm{d} z) \leq C(T) .
$$

It follows from (21), (22) and (23) that

$$
\begin{aligned}
I_{2} & =\int_{\mathbb{H}}\left|\frac{\varphi(z, \varepsilon V(t))-1}{\varepsilon}\right|^{2} \rho(z) \mu(\mathrm{d} z) \\
& \leq C(T) I_{3}+C(T) I_{4} \leq C(T) .
\end{aligned}
$$

By (11) and triangle inequality, we have

$$
\begin{aligned}
\int_{\mathbb{H}} & \left|\frac{\lambda^{\varepsilon}(t, z)-1}{\varepsilon}\right|^{2} \rho(z) \mu(\mathrm{d} z) \\
& =\frac{1}{\varepsilon^{2}} \int_{\mathbb{H}}\left|\varphi(z, \varepsilon V(t)) \frac{\rho(z+\varepsilon V(t))}{\rho(z)}-1\right|^{2} \rho(z) \mu(\mathrm{d} z) \\
& \leq 2 I_{1}+2 I_{2} \leq C(T) .
\end{aligned}
$$


Combining this with (13) and Burkholder's inequality, we have

$$
\begin{aligned}
\mathbb{E}\left\{\sup _{t \leq T}\left|\frac{\Theta_{t}^{\varepsilon}-1}{\varepsilon}\right|^{2}\right\} \\
=\mathbb{E}\left\{\sup _{t \leq T}\left|\int_{0}^{t} \int_{\mathbb{H}} \frac{\Theta_{s-}^{\varepsilon}\left(\lambda^{\varepsilon}(s, z)-1\right)}{\varepsilon} \tilde{N}(\mathrm{~d} z, \mathrm{~d} s)\right|^{2}\right\} \\
\leq C(T) \mathbb{E}\left\{\int_{0}^{T} \int_{\mathbb{H}} \sup _{s \leq t}\left|\frac{\Theta_{s}^{\varepsilon}-1}{\varepsilon}\right|^{2}\left|\lambda^{\varepsilon}(t, z)-1\right|^{2} \rho(z) \mu(\mathrm{d} z) \mathrm{d} t\right\} \\
\quad+C(T) \mathbb{E}\left\{\int_{0}^{T} \int_{\mathbb{H}}\left|\frac{\lambda^{\varepsilon}(t, z)-1}{\varepsilon}\right|^{2} \rho(z) \mu(\mathrm{d} z) \mathrm{d} t\right\} \\
\leq C(T) \int_{0}^{T} \mathbb{E} \sup _{s \leq t}\left|\frac{\Theta_{s}^{\varepsilon}-1}{\varepsilon}\right|^{2} \mathrm{~d} t+C(T) .
\end{aligned}
$$

Then Gronwall's inequality implies

$$
\mathbb{E}\left\{\sup _{0 \leq t \leq T}\left|\frac{\Theta_{t}^{\varepsilon}-1}{\varepsilon}\right|^{2}\right\} \leq C(T) .
$$

Furthermore, we have

$$
\sup _{\varepsilon<1} \mathbb{E}\left\{\sup _{0 \leq t \leq T}\left|\frac{\Theta_{t}^{\varepsilon}-1}{\varepsilon}\right|^{2}\right\}<\infty
$$

2.2. Malliavin derivatives. Let $G_{t}:=G\left(\{N(\mathrm{~d} z, \mathrm{~d} s)\}_{s \leq t}\right)$ be an $\mathbb{H}$-valued functional. Denote $G_{t}^{\varepsilon}:=G\left(\left\{N^{\varepsilon}(\mathrm{d} z, \mathrm{~d} s)\right\}_{s \leq t}\right)$, where $N^{\varepsilon}$ is defined in (10).

Definition 5. A Poisson functional $G_{t}$ is called to be Malliavin differentiable along some $V \in \mathbb{V}$, if for some $p \geq 1$ there exists a random variable denoted by $D_{V} G_{t}$ with $\mathbb{E}\left|D_{V} G_{t}\right|^{p}<\infty$, such that

$$
\lim _{\varepsilon \rightarrow 0} \mathbb{E}\left|\varepsilon^{-1}\left(G_{t}^{\varepsilon}-G_{t}\right)-D_{V} G_{t}\right|^{p}=0 \text {. }
$$

REMARK 3. This notion appeared firstly in [4]. It is based on Bismut's approach about the Malliavin calculus with jumps in [6].

Before we move on, it is necessary for us to prove the Malliavin differentiability of the solution to (2).

Proposition 6. Let $\left(\mathbf{H}_{\nu}\right)$ hold. Assume A generates the $C_{0}$-semigroup $\{S(t)\}_{t \geq 0}$ and $F \in C_{b}^{2}(\mathbb{H}, \mathbb{H})$. Then for any $V \in \mathbb{V}, X_{t}$ is Malliavin differentiable along $V$. Moreover, the derivative satisfies the following equation:

$$
\left\{\begin{array}{l}
\mathrm{d} D_{V} X_{t}=A D_{V} X_{t} \mathrm{~d} t+\nabla F\left(X_{t}\right) D_{V} X_{t} \mathrm{~d} t+\int_{\mathbb{H}} V(t) N(\mathrm{~d} z, \mathrm{~d} t) \\
D_{V} X_{0}=0
\end{array}\right.
$$


Proof. It is easy to prove that there exists a unique solution to the linear equation (27). And the solution can be written as

$$
\begin{aligned}
D_{V} X_{t}= & \int_{0}^{t} S(t-s) \nabla F\left(X_{s}\right) D_{V} X_{s} \mathrm{~d} s \\
& +\int_{0}^{t} \int_{\mathbb{H}} S(t-s) V(s) N(\mathrm{~d} z, \mathrm{~d} s) .
\end{aligned}
$$

For each $\varepsilon>0$, let $L^{\varepsilon}$ be the perturbed process of $L$,

$$
L_{t}^{\varepsilon}:=\int_{0}^{t} \int_{\mathbb{H}} z N^{\varepsilon}(\mathrm{d} z, \mathrm{~d} s), \quad \forall t \in[0, T] .
$$

Then by (10) we have

$$
L_{t}^{\varepsilon}=L_{t}+\varepsilon \int_{0}^{t} \int_{\mathbb{H}} V(s) N(\mathrm{~d} z, \mathrm{~d} s), \quad \forall t \in[0, T] .
$$

Let $\left\{X_{t}^{\varepsilon}\right\}_{t \leq T}$ be the solution of the following equation:

$$
X_{t}^{\varepsilon}=x+\int_{0}^{t} S(t-s) F\left(X_{s}^{\varepsilon}\right) \mathrm{d} s+\int_{0}^{t} S(t-s) \mathrm{d} L_{s}^{\varepsilon}+\int_{0}^{t} S(t-s) \mathrm{d} Z_{s} .
$$

Now we aim to prove

$$
\lim _{\varepsilon \rightarrow 0} \mathbb{E}\left\{\sup _{s \leq t}\left|\frac{X_{s}^{\varepsilon}-X_{s}}{\varepsilon}-D_{V} X_{s}\right|\right\}=0
$$

In fact, it follows (3), (29) and (30) that

$$
\begin{aligned}
X_{t}^{\varepsilon}-X_{t}= & \int_{0}^{t} S(t-s)\left(F\left(X_{s}^{\varepsilon}\right)-F\left(X_{s}\right)\right) \mathrm{d} s \\
& +\varepsilon \int_{0}^{t} \int_{\mathbb{H}} S(t-s) V(s) N(\mathrm{~d} z, \mathrm{~d} s) .
\end{aligned}
$$

Since $A$ is a generator of $C_{0}$-semigroup on $\mathbb{H}$, then there exist constant $C_{A}>0$ and $\kappa>0$ such that $\|S(t)\| \leq C_{A} e^{\kappa t}$ for $t \in[0, T]$. Therefore,

$$
\begin{aligned}
\left|X_{t}^{\varepsilon}-X_{t}\right| \leq & \int_{0}^{t}\left|S(t-s)\left(F\left(X_{s}^{\varepsilon}\right)-F\left(X_{s}\right)\right)\right| \mathrm{d} s \\
& +\varepsilon \int_{0}^{t} \int_{\mathbb{H}}|S(t-s) V(s)| N(\mathrm{~d} z, \mathrm{~d} s) \\
\leq & C_{A}\|\nabla F\|_{\infty} \int_{0}^{t} e^{\kappa(t-s)}\left|X_{s}^{\varepsilon}-X_{s}\right| \mathrm{d} s \\
& +\varepsilon C_{A} \int_{0}^{t} \int_{\mathbb{H}} e^{\kappa(t-s)}|V(s)| N(\mathrm{~d} z, \mathrm{~d} s) .
\end{aligned}
$$


Then,

$$
\begin{aligned}
\sup _{s \leq t}\left\{e^{-\kappa s}\left|X_{s}^{\varepsilon}-X_{s}\right|\right\} \leq & C_{A}\|\nabla F\|_{\infty} \int_{0}^{t} \sup _{r \leq s}\left\{e^{-\kappa r}\left|X_{r}^{\varepsilon}-X_{r}\right|\right\} \mathrm{d} s \\
& +\varepsilon C_{A} \int_{0}^{t} \int_{\mathbb{H}} e^{-\kappa s}|V(s)| N(\mathrm{~d} z, \mathrm{~d} s) .
\end{aligned}
$$

By Gronwall's inequality, we have

(33) $\sup _{s \leq t}\left|X_{s}^{\varepsilon}-X_{s}\right| \leq \varepsilon C_{A} \exp \left\{\kappa t+C_{A}\|\nabla F\|_{\infty} t\right\} \int_{0}^{t} \int_{\mathbb{H}}|V(s)| N(\mathrm{~d} z, \mathrm{~d} s)$.

By (28), (32) and Taylor's formula, we arrive at

$$
\begin{aligned}
& \left|\frac{1}{\varepsilon}\left(X_{t}^{\varepsilon}-X_{t}\right)-D_{V} X_{t}\right| \\
& \quad \leq C_{A} \int_{0}^{t} e^{\kappa(t-s)}\left|\frac{1}{\varepsilon}\left(F\left(X_{s}^{\varepsilon}\right)-F\left(X_{s}\right)\right)-\nabla F\left(X_{s}\right) D_{V} X_{s}\right| \mathrm{d} s \\
& \leq C_{A} \int_{0}^{t} e^{\kappa(t-s)}\left\{\|\nabla F\|_{\infty}\left|\frac{1}{\varepsilon}\left(X_{s}^{\varepsilon}-X_{s}\right)-D_{V} X_{s}\right|\right. \\
& \left.\quad+\frac{1}{\varepsilon}\left\|\nabla^{2} F\right\|_{\infty}\left|X_{s}^{\varepsilon}-X_{s}\right|^{2}\right\} \mathrm{d} s .
\end{aligned}
$$

Gronwall's inequality implies

$$
\begin{aligned}
& \sup _{s \leq t}\left|\frac{1}{\varepsilon}\left(X_{s}^{\varepsilon}-X_{s}\right)-D_{V} X_{s}\right| \\
& \quad \leq C_{A}\left\|\nabla^{2} F\right\|_{\infty} t \exp \left\{C\|\nabla F\|_{\infty} t+\kappa t\right\} \frac{1}{\varepsilon} \sup _{s \leq t}\left|X_{s}^{\varepsilon}-X_{s}\right|^{2} .
\end{aligned}
$$

Combining this with (33), we can obtain

$$
\mathbb{E}\left\{\sup _{s \leq t}\left|\frac{1}{\varepsilon}\left(X_{s}^{\varepsilon}-X_{s}\right)-D_{V} X_{s}\right|\right\} \leq C_{1} \mathbb{E}\left\{\int_{0}^{t} \int_{\mathbb{H}}|V(s)| N(\mathrm{~d} z, \mathrm{~d} s)\right\}^{2} \varepsilon,
$$

where $C_{1}$ is a constant independent of $\varepsilon$. In view of $V \in \mathbb{V}$ and $\nu$ is a finite measure, we have

$$
\begin{aligned}
& \mathbb{E}\left\{\int_{0}^{t} \int_{\mathbb{H}}|V(s)| N(\mathrm{~d} z, \mathrm{~d} s)\right\}^{2} \\
& \quad \leq 2 \nu(\mathbb{H}) \int_{0}^{t} \mathbb{E} V^{2}(s) \mathrm{d} s+2 \nu(\mathbb{H})^{2} \mathbb{E}\left(\int_{0}^{t} \mathbb{E} V(s) \mathrm{d} s\right)^{2} \\
& \quad \leq 2\left(\nu(\mathbb{H})+\nu(\mathbb{H})^{2} T\right) \int_{0}^{t} \mathbb{E} V^{2}(s) \mathrm{d} s<\infty
\end{aligned}
$$


Therefore, there exists a constant $C_{2}>0$ such that

$$
\mathbb{E}\left\{\sup _{s \leq t}\left|\frac{1}{\varepsilon}\left(X_{s}^{\varepsilon}-X_{s}\right)-D_{V} X_{s}\right|\right\} \leq C_{2} \varepsilon
$$

which yields (31).

Proposition 7 (Chain rule). Let $\left(\mathbf{H}_{\nu}\right)$ hold. Assume $A$ generates a $C_{0}$ semigroup $\{S(t)\}_{t \geq 0}$ and $F \in C_{b}^{2}(\mathbb{H}, \mathbb{H})$. Then for any $f \in C_{b}^{2}(\mathbb{H})$ and $V \in \mathbb{V}$, $f\left(X_{t}\right)$ is Malliavin differentiable and

$$
D_{V} f\left(X_{t}\right)=\left\langle\nabla f\left(X_{t}\right), D_{V} X_{t}\right\rangle .
$$

Proof. In view of Proposition 6, we have

$$
\mathbb{E}\left|\left\langle\nabla f\left(X_{t}\right), D_{V} X_{t}\right\rangle\right| \leq\|\nabla f\|_{\infty} \mathbb{E}\left|D_{V} X_{t}\right|<\infty
$$

Meanwhile, by Taylor's formula we obtain

$$
\begin{aligned}
& \left|\frac{1}{\varepsilon}\left(f\left(X_{t}^{\varepsilon}\right)-f\left(X_{t}\right)\right)-\left\langle\nabla f\left(X_{t}\right), D_{V} X_{t}\right\rangle\right| \\
& \quad \leq\|\nabla f\|_{\infty}\left|\frac{1}{\varepsilon}\left(X_{t}^{\varepsilon}-X_{t}\right)-D_{V} X_{t}\right|+\frac{1}{2 \varepsilon}\left\|\nabla^{2} f\right\|_{\infty}\left|X_{t}^{\varepsilon}-X_{t}\right|^{2} .
\end{aligned}
$$

Combining this with (33) and (34), we arrive at

$$
\lim _{\varepsilon \rightarrow 0} \mathbb{E}\left|\frac{1}{\varepsilon}\left(f\left(X_{t}^{\varepsilon}\right)-f\left(X_{t}\right)\right)-\left\langle\nabla f\left(X_{t}\right), D_{V} X_{t}\right\rangle\right|=0 .
$$

2.3. Integration by parts formula. Now we are ready to give the following integration by parts formula.

THEOREM 8. Let $\left(\mathbf{H}_{\nu}\right)$ hold. Assume the operator A generates a strongly continuous semigroups $\{S(t)\}_{t \geq 0}$ and $F \in C_{b}^{2}(\mathbb{H}, \mathbb{H})$. Then for any $f \in C_{b}^{2}(\mathbb{H})$ and $V \in \mathbb{V}$, we have

$$
\mathbb{E}\left\{D_{V} f\left(X_{t}\right)\right\}=-\mathbb{E}\left\{f\left(X_{t}\right) M_{t}\right\}, \quad t \leq T,
$$

with

$$
M_{t}=\int_{0}^{t} \int_{\mathbb{H}}\left(\left\langle z, Q^{-1} V(s)\right\rangle+\langle\nabla \log \rho(z), V(s)\rangle\right) \tilde{N}(\mathrm{~d} z, \mathrm{~d} s) .
$$

REMARK 4. From the proof below, we will see that the formula hold for Poisson functionals which are Malliavin differentiable along some $V \in \mathbb{V}$.

Proof. We give the proof in three steps.

Step 1. Assume $V \in \mathbb{V}_{0}$ and $\rho \geq \delta$ for some $\delta>0$. By Lemma 3, for any $\varepsilon \in(0,1)$, we have

$$
\mathbb{E} f\left(X_{t}\right)=\mathbb{E}\left\{f\left(X_{t}^{\varepsilon}\right) \Theta_{t}^{\varepsilon}\right\} .
$$

Then,

$$
\frac{1}{\varepsilon} \mathbb{E}\left(f\left(X_{t}^{\varepsilon}\right) \Theta_{t}^{\varepsilon}-f\left(X_{t}\right)\right)=0 .
$$


Furthermore,

$$
\frac{1}{\varepsilon} \mathbb{E}\left(f\left(X_{t}^{\varepsilon}\right)-f\left(X_{t}\right)\right)+\frac{1}{\varepsilon} \mathbb{E} f\left(X_{t}^{\varepsilon}\right)\left(\Theta_{t}^{\varepsilon}-1-\varepsilon M_{t}\right)+\mathbb{E} f\left(X_{t}^{\varepsilon}\right) M_{t}=0,
$$

where

$$
M_{t}=\int_{0}^{t} \int_{\mathbb{H}}\left(\left\langle z, Q^{-1} V(s)\right\rangle+\langle\nabla \log \rho(z), V(s)\rangle\right) \tilde{N}(\mathrm{~d} z, \mathrm{~d} s) .
$$

For the first and third terms of (37), by Proposition 7, we obtain

$$
\begin{aligned}
\lim _{\varepsilon \rightarrow 0} \frac{1}{\varepsilon} \mathbb{E}\left(f\left(X_{t}^{\varepsilon}\right)-f\left(X_{t}\right)\right) & =\mathbb{E} D_{V} f\left(X_{t}\right), \\
\lim _{\varepsilon \rightarrow 0} \mathbb{E}\left\{f\left(X_{t}^{\varepsilon}\right) M_{t}\right\} & =\mathbb{E}\left\{f\left(X_{t}\right) M_{t}\right\} .
\end{aligned}
$$

It follows from (11) and (12) that

$$
\lim _{\varepsilon \rightarrow 0} \Theta_{s}^{\varepsilon}=1, \quad \forall s \in(0, T] .
$$

Observe that $\nu$ is a finite measure, then also by (11) and (12) we arrive at

$$
\begin{aligned}
\lim _{\varepsilon \rightarrow 0} \frac{1}{\varepsilon}\left(\Theta_{t}^{\varepsilon}-1\right) & =\lim _{\varepsilon \rightarrow 0} \frac{1}{\varepsilon} \int_{0}^{t} \int_{\mathbb{H}} \Theta_{s-}^{\varepsilon}\left(\lambda^{\varepsilon}(s, z)-1\right) \tilde{N}(\mathrm{~d} z, \mathrm{~d} s) \\
& =\int_{0}^{t} \int_{\mathbb{H}} \lim _{\varepsilon \rightarrow 0} \Theta_{s-\frac{1}{\varepsilon}}^{\varepsilon}\left(\lambda^{\varepsilon}(s, z)-1\right) \tilde{N}(\mathrm{~d} z, \mathrm{~d} s) \\
& =\int_{0}^{t} \int_{\mathbb{H}} \lim _{\varepsilon \rightarrow 0} \frac{1}{\varepsilon}\left(\lambda^{\varepsilon}(s, z)-1\right) \tilde{N}(\mathrm{~d} z, \mathrm{~d} s) \\
& =\int_{0}^{t} \int_{\mathbb{H}}\left(\left\langle z, Q^{-1} V(s)\right\rangle+\langle\nabla \log \rho(z), V(s)\rangle\right) \tilde{N}(\mathrm{~d} z, \mathrm{~d} s)
\end{aligned}
$$

Combining this with Lemma 4, we obtain

$$
\lim _{\varepsilon \rightarrow 0} \frac{1}{\varepsilon} \mathbb{E}\left|\Theta_{t}^{\varepsilon}-1-\varepsilon M_{t}\right|=0 \text {. }
$$

Then

$$
\lim _{\varepsilon \rightarrow 0} \frac{1}{\varepsilon}\left|\mathbb{E} f\left(X_{t}\right)\left(\Theta_{t}^{\varepsilon}-1-\varepsilon M_{t}\right)\right| \leq\|f\|_{\infty} \lim _{\varepsilon \rightarrow 0} \frac{1}{\varepsilon} \mathbb{E}\left|\Theta_{t}^{\varepsilon}-1-\varepsilon M_{t}\right|=0
$$

Letting $\varepsilon \rightarrow 0$ in (37), by (38) and (39), we derive (36).

Step 2. Assume $V \in \mathbb{V}_{0}$. For $n \geq 1$, let $L^{n}:=\left\{L_{t}^{n}\right\}_{t \geq 0}$ be a purely jump Lévy process with characteristic measure $\frac{1}{n} \mu$ and jump measure $N^{n}(\mathrm{~d} z, \mathrm{~d} t)$. Assume that $L^{n}, L$ and $Z$ are independent. Now, $L+L^{n}$ is a jump process with Lévy measure $\nu+\frac{1}{n} \mu$. Moreover, its jump measure is $N_{n}(\mathrm{~d} z, \mathrm{~d} t):=$ $N(\mathrm{~d} z, \mathrm{~d} t)+N^{n}(\mathrm{~d} z, \mathrm{~d} t)$. Let $\widetilde{N}_{n}(\mathrm{~d} z, \mathrm{~d} t)$ be the associated martingale measure, that is, $\widetilde{N}_{n}(\mathrm{~d} z, \mathrm{~d} t):=N_{n}(\mathrm{~d} z, \mathrm{~d} t)-\left(\rho(z)+\frac{1}{n}\right) \mu(\mathrm{d} z) \mathrm{d} t$. Let $D_{V}^{n}$ be the derivative operator associated with $N_{n}(\mathrm{~d} z, \mathrm{~d} t)$, which is defined as in Definition 5 . 
Let $\left\{X_{t}^{n}\right\}_{t \leq T}$ be the solution to the following equation:

$$
\left\{\begin{array}{l}
\mathrm{d} X_{t}^{n}=A X_{t}^{n} \mathrm{~d} t+F\left(X_{t}^{n}\right) \mathrm{d} t+\mathrm{d} L_{t}+\mathrm{d} L_{t}^{n}+\mathrm{d} Z_{t}, \\
X_{0}=x .
\end{array}\right.
$$

Then it is easy to see that

$$
\lim _{n \rightarrow \infty} \mathbb{E}\left\{\sup _{s \leq t}\left|X_{s}^{n}-X_{s}\right|^{2}\right\}=0, \quad \forall t \in[0, T] .
$$

According to Proposition 6, the derivative $D_{V}^{n} X_{t}^{n}$ exists and satisfies

$$
D_{V}^{n} X_{t}^{n}=\int_{0}^{t} S(t-s) \nabla F\left(X_{s}^{n}\right) D_{V}^{n} X_{s}^{n} \mathrm{~d} s+\int_{0}^{t} \int_{\mathbb{H}} V(s) N_{n}(\mathrm{~d} z, \mathrm{~d} s) .
$$

Moreover, we have $\mathbb{E}\left\{\sup _{s \leq t}\left|D_{V}^{n} X_{s}\right|^{2}\right\}<\infty$. By Step 1,

$$
\mathbb{E} D_{V}^{n} f\left(X_{t}^{n}\right)=-\mathbb{E}\left\{f\left(X_{t}^{n}\right) M_{t}^{n}\right\},
$$

where

$$
M_{t}^{n}=\int_{0}^{t} \int_{\mathbb{H}}\left(\left\langle z, Q^{-1} V(s)\right\rangle+\left\langle\frac{\nabla \rho(z)}{\rho(z)+\frac{1}{n}}, V(s)\right\rangle\right) \widetilde{N_{n}}(\mathrm{~d} z, \mathrm{~d} s) .
$$

Then it follows from (28) and (42) that

$$
\begin{aligned}
& \mathbb{E}\left\{\sup _{s \leq t}\left|D_{V}^{n} X_{s}-D_{V} X_{s}\right|\right\} \\
& \leq \mathbb{E} \int_{0}^{t}\left|S(t-s) \nabla F\left(X_{s}^{n}\right) D_{V}^{n} X_{s}^{n}-S(t-s) \nabla F\left(X_{s}\right) D_{V} X_{s}\right| \mathrm{d} s \\
&+\mathbb{E} \int_{0}^{t}|S(t-s) V(s)| N^{n}(\mathrm{~d} z, \mathrm{~d} s) \\
& \leq \mathbb{E} \int_{0}^{t}\left|S(t-s) \nabla F\left(X_{s}^{n}\right) D_{V}^{n} X_{s}^{n}-S(t-s) \nabla F\left(X_{s}^{n}\right) D_{V} X_{s}\right| \mathrm{d} s+\frac{C(t)}{n} \\
&+\mathbb{E} \int_{0}^{t}\left|S(t-s) \nabla F\left(X_{s}^{n}\right) D_{V} X_{s}-S(t-s) \nabla F\left(X_{s}\right) D_{V} X_{s}\right| \mathrm{d} s \\
& \leq C(t) \int_{0}^{t} \mathbb{E}\left(\sup _{r \leq s}\left|D_{V}^{n} X_{r}^{n}-D_{V} X_{r}\right|\right) \mathrm{d} s+\frac{C(t)}{n} \\
&+C(t)\left(\mathbb{E} \sup _{s \leq t}\left|X_{s}^{n}-X_{s}\right|^{2}\right)^{\frac{1}{2}},
\end{aligned}
$$

where $C(t)$ is a generic constant independent of $n$. Gronwall's inequality yields

$$
\begin{aligned}
& \lim _{n \rightarrow \infty} \mathbb{E}\left\{\sup _{s \leq t}\left|D_{V}^{n} X_{s}-D_{V} X_{s}\right|\right\} \\
& \leq C(t) \lim _{n \rightarrow \infty}\left\{\frac{1}{n}+\left(\mathbb{E} \sup _{s \leq t}\left|X_{s}^{n}-X_{s}\right|^{2}\right)^{\frac{1}{2}}\right\}=0 .
\end{aligned}
$$


By Proposition 7, the triangle inequality and Hörder inequality, one can derive

$$
\begin{aligned}
\lim _{n \rightarrow \infty} \mid & \mathbb{E} D_{V}^{n} f\left(X_{t}^{n}\right)-\mathbb{E} D_{V} f\left(X_{t}\right) \mid \\
\leq & \lim _{n \rightarrow \infty} \mathbb{E}\left|\left\langle\nabla f\left(X_{t}^{n}\right), D_{V}^{n} X_{t}\right\rangle-\left\langle\nabla f\left(X_{t}\right), D_{V} X_{t}\right\rangle\right| \\
\leq & \lim _{n \rightarrow \infty} \mathbb{E}\left|\left\langle\nabla f\left(X_{t}^{n}\right), D_{V}^{n} X_{t}\right\rangle-\left\langle\nabla f\left(X_{t}^{n}\right), D_{V} X_{t}\right\rangle\right| \\
& +\lim _{n \rightarrow \infty} \mathbb{E}\left|\left\langle\nabla f\left(X_{t}^{n}\right), D_{V} X_{t}\right\rangle-\left\langle\nabla f\left(X_{t}\right), D_{V} X_{t}\right\rangle\right| \\
\leq & \|\nabla f\|_{\infty} \lim _{n \rightarrow \infty} \mathbb{E}\left|D_{V}^{n} X_{t}^{n}-D_{V} X_{t}\right| \\
& +\left\|\nabla^{2} f\right\|_{\infty}\left\{\mathbb{E}\left|D_{V} X_{t}\right|^{2}\right\}^{\frac{1}{2}} \lim _{n \rightarrow \infty}\left\{\mathbb{E}\left|X_{t}^{n}-X_{t}\right|^{2}\right\}^{\frac{1}{2}}=0 .
\end{aligned}
$$

Meanwhile, by the same argument, one can also have

$$
\lim _{n \rightarrow \infty} \mathbb{E}\left\{f\left(X_{t}^{n}\right) M_{t}^{n}\right\}=\mathbb{E}\left\{f\left(X_{t}\right) M_{t}\right\} .
$$

Now, using (45), (46) and letting $n \rightarrow \infty$ in (43), we obtain (36).

Step 3. Assume $V \in \mathbb{V}$. For $n \geq 1$, define

$$
V_{n}(t)=V(t) I_{[0, n]}\left(\left|Q^{-1} V(t)\right|\right), \quad t \in[0, T] .
$$

Then $V_{n} \in \mathbb{V}_{0}$. By Step 2, we have

$$
\mathbb{E} D_{V_{n}} f\left(X_{t}\right)=-\mathbb{E}\left\{f\left(X_{t}\right) M_{t}^{(n)}\right\},
$$

with

$$
M_{t}^{(n)}=\int_{0}^{t} \int_{\mathbb{H}}\left(\left\langle z, Q^{-1} V_{n}(s)\right\rangle+\left\langle\nabla \log \rho(z), V_{n}(s)\right\rangle\right) \tilde{N}(\mathrm{~d} z, \mathrm{~d} s) .
$$

It is easy to check that

$$
\lim _{n \rightarrow \infty} \mathbb{E}\left|M_{t}^{n}-M_{t}\right|=0
$$

Meanwhile, observe that

$$
\begin{aligned}
\mathbb{E}\left|D_{V_{n}} X_{t}-D_{V} X_{t}\right| \leq & \mathbb{E} \int_{0}^{t}\left|S(t-s) \nabla F\left(X_{s}\right)\left(D_{V_{n}} X_{s}-D_{V} X_{s}\right)\right| \mathrm{d} s \\
& +\mathbb{E} \int_{0}^{t} \int_{\mathbb{H}}\left|S(t-s)\left(V_{n}(s)-V(s)\right)\right| N(\mathrm{~d} z, \mathrm{~d} s) \\
\leq & C_{A}\|\nabla F\|_{\infty} \int_{0}^{t} e^{(t-s) \kappa} \mathbb{E}\left|D_{V_{n}} X_{s}-D_{V} X_{s}\right| \mathrm{d} s \\
& +C_{A} \lambda \int_{0}^{t} e^{(t-s) \kappa} \mathbb{E}\left|V_{n}(s)-V(s)\right| \mathrm{d} s .
\end{aligned}
$$

Gronwall's inequality yields

$$
\begin{aligned}
& \mathbb{E}\left|D_{V_{n}} X_{t}-D_{V} X_{t}\right| \\
& \quad \leq C_{A} \lambda \exp \left\{\left(C_{A}\|\nabla F\|_{\infty}+\kappa\right) t\right\} \int_{0}^{t} \mathbb{E}\left|V_{n}(s)-V(s)\right| \mathrm{d} s,
\end{aligned}
$$


which tends to 0 as $n \rightarrow \infty$. Therefore,

$$
\begin{aligned}
& \lim _{n \rightarrow \infty}\left|\mathbb{E} D_{V_{n}} f\left(X_{t}\right)-\mathbb{E} D_{V} f\left(X_{t}\right)\right| \\
& \quad \leq\|\nabla f\|_{\infty} \lim _{n \rightarrow \infty} \mathbb{E}\left|D_{V_{n}} X_{t}-D_{V} X_{t}\right|=0 .
\end{aligned}
$$

With the help of (48) and (49), we finish the proof by letting $n \rightarrow \infty$ in (47).

\section{Proofs of main results}

We use the notation $J_{s t}$ with $s \leq t$ for the derivative flow between times $s$ and $t$, that is, for every $\xi \in \mathbb{H}, J_{s t} \xi$ is the solution of

$$
\left\{\begin{array}{l}
\mathrm{d} J_{s t} \xi=A J_{s t} \xi \mathrm{d} t+\nabla F\left(X_{t}\right) J_{s t} \xi \mathrm{d} t, \\
J_{s s} \xi=\xi .
\end{array}\right.
$$

Note that we have the important cocycle property $J_{s t}=J_{r t} J_{s r}$ for $r \in[s, t]$. By (27) and (50), we can derive

$$
D_{V} X_{t}=\int_{0}^{t} \int_{\mathbb{H}} J_{s t} V(s) N(\mathrm{~d} z, \mathrm{~d} s) .
$$

In the following discussions, for the sake of writing, let us denote $J_{t}:=J_{0 t}$. To summarize, $J_{t} \xi$ is the effect on $X_{t}$ of an infinitesimal perturbation of the initial condition along the direction $\xi$ and $D_{V} X_{t}$ is the effect on $X_{t}$ of an infinitesimal perturbation of the Poisson jump measure $N(\mathrm{~d} z, \mathrm{~d} t)$ along the direction $V$.

3.1. Proof of Theorem 1. We will give the proof in two steps.

Step1: Let us first assume $F \in C_{b}^{2}(\mathbb{H}, \mathbb{H})$. For each $\xi \in \mathbb{H}$, by $(50)$ we have

$$
J_{t} \xi=S(t) \xi+\int_{0}^{t} S(t-s) \nabla F\left(X_{s}\right) J_{s} \xi \mathrm{d} s .
$$

In view of $\left(\mathbf{H}_{A}\right)$, we have $\|S(t)\| \leq e^{-\gamma_{1} t}$ for each $t \geq 0$. Then

$$
\left|J_{t} \xi\right| \leq e^{-\gamma_{1} t}|\xi|+\|\nabla F\|_{\infty} \int_{0}^{t} e^{-\gamma_{1}(t-s)}\left|J_{s} \xi\right| \mathrm{d} s .
$$

Gronwall's inequality implies

$$
\left|J_{t} \xi\right| \leq \exp \left\{\left(-\gamma_{1}+\|\nabla F\|_{\infty}\right) t\right\}|\xi| .
$$

Observe that

$$
\begin{aligned}
Q^{-1} J_{t} \xi & =Q^{-1} S(t) \xi+Q^{-1} \int_{0}^{t} S(t-s) \nabla F\left(X_{s}\right) J_{s} \xi \mathrm{d} s \\
& =Q^{-1} S(t) \xi+\int_{0}^{t} Q^{-1} S(t-s) \nabla F\left(X_{s}\right) J_{s} \xi \mathrm{d} s
\end{aligned}
$$


where we use $\left(\mathbf{H}_{S Q}\right)$ in the second equality. Then one has

$$
\begin{aligned}
\int_{0}^{t}\left|Q^{-1} J_{s} \xi\right| \mathrm{d} s \leq & \int_{0}^{t}\left|Q^{-1} S(s) \xi\right| \mathrm{d} s \\
& +\|\nabla F\|_{\infty} \int_{0}^{t} \int_{0}^{s}\left\|Q^{-1} S(s-r)\right\||| J_{r} \xi \mid \mathrm{d} r \mathrm{~d} s \\
\leq & \left(1+\exp \left\{\left(-\gamma_{1}+\|\nabla F\|_{\infty}\right) t\right\} t\right)|\xi| \int_{0}^{t}\left\|Q^{-1} S(s)\right\| \mathrm{d} s<\infty
\end{aligned}
$$

which together with (53) show $\left\{J_{s} \xi\right\}_{s \leq T} \in \mathbb{V}$. Now set $V(s)=J_{s} \xi$ in (51). Then we obtain $D_{V} X_{t}=N_{t} J_{t} \xi$ with $N_{t}:=N([0, t] \times \mathbb{H})$. Therefore,

$$
\frac{I_{\left[N_{t} \geq 1\right]}}{N_{t}} D_{V} X_{t}=J_{t} \xi I_{\left[N_{t} \geq 1\right]},
$$

where $I_{\left[N_{t} \geq 1\right]}$ is an indicator function and we let $\frac{0}{0}=0$ for convention. Let $\beta:[0, \infty) \rightarrow[0, \infty)$ be a smooth function satisfying $\beta(y)=y^{2}, y \in\left[0, \frac{1}{2}\right]$ and $\beta(y) \equiv 1, y \in[1, \infty)$. By Proposition 7 and the fact $D_{V} N_{t}=0$, we have

$$
D_{V}\left\{\frac{I_{\left[N_{t} \geq 1\right]}}{N_{t}}\right\}=D_{V}\left\{\frac{\beta\left(N_{t}\right)}{N_{t}}\right\}=\frac{\beta^{\prime}\left(N_{t}\right) N_{t}-\beta\left(N_{t}\right)}{N_{t}^{2}} D_{V} N_{t}=0 .
$$

It follows from (55), (56) and Theorem 8 that

$$
\begin{aligned}
\nabla_{\xi} P_{t}^{1} f(x)= & \nabla_{\xi} \mathbb{E}\left\{f\left(X_{t}^{x}\right) I_{\left[N_{t} \geq 1\right]}\right\} \\
= & \mathbb{E}\left\langle\nabla f\left(X_{t}^{x}\right), J_{t} \xi I_{\left[N_{t} \geq 1\right]}\right\rangle \\
= & \mathbb{E}\left\langle\nabla f\left(X_{t}^{x}\right), \frac{I_{\left[N_{t} \geq 1\right]}}{N_{t}} D_{V} X_{t}^{x}\right\rangle \\
= & \mathbb{E}\left\{D_{V} f\left(X_{t}^{x}\right) \frac{I_{\left[N_{t} \geq 1\right]}}{N_{t}}\right\} \\
= & \mathbb{E}\left\{D_{V}\left(f\left(X_{t}^{x}\right) \frac{I_{\left[N_{t} \geq 1\right]}}{N_{t}}\right)\right\} \\
= & -\mathbb{E}\left\{f\left(X_{t}^{x}\right) \frac{I_{\left[N_{t} \geq 1\right]}}{N_{t}}\right. \\
& \left.\times \int_{0}^{t} \int_{\mathbb{H}}\left(\left\langle z, Q^{-1} J_{s} \xi\right\rangle+\left\langle\nabla \log \rho(z), J_{s} \xi\right\rangle\right) \tilde{N}(\mathrm{~d} z, \mathrm{~d} s)\right\} .
\end{aligned}
$$

Step 2: Assume $F \in C_{b}^{1}(\mathbb{H}, \mathbb{H})$ and $\nabla F$ is Lipschitz continuous. We aim to construct approximation sequence $\left\{F_{k}\right\}_{k \geq 1} \subset C_{b}^{2}(\mathbb{H}, \mathbb{H})$ such that $F_{k} \rightarrow F$ and $\nabla F_{k} \rightarrow \nabla F$ in pointwise sense as $k \rightarrow \infty$. In fact, for $k \geq 1$, we take a sequence of non-negative, twice differential function $\left\{g_{k}\right\}_{k \geq 1}$ such that

$$
\operatorname{Supp}\left\{g_{k}\right\} \subset\left\{y \in \mathbb{R}^{k}:|y|_{\mathbb{R}^{k}} \leq \frac{1}{k}\right\}, \quad \int_{\mathbb{R}^{k}} g_{k}(y) \mathrm{d} y=1 .
$$


Identifying $\mathbb{R}^{k}$ with $\operatorname{span}\left\{e_{1}, \ldots, e_{k}\right\}$, we define

$$
F_{k}(x)=\int_{\mathbb{R}^{k}} g_{k}\left(y-\Pi_{k} x\right) F\left(\sum_{i=1}^{k} y_{i} e_{i}\right) \mathrm{d} y,
$$

where $\Pi_{k}: \mathbb{H} \rightarrow \operatorname{span}\left\{e_{1}, \ldots, e_{k}\right\}$ is the projection operator. Then $F_{k}$ is a twice differentiable function with bounded and continuous derivatives (see [10, P127]). Furthermore, we have $\sup _{k>1}\left\|\nabla F_{k}\right\|_{\infty} \leq\|\nabla F\|_{\infty}$ and $\sup _{k \geq 1}\left\|\nabla^{2} F_{k}\right\|_{\infty} \leq\|\nabla F\|_{\text {Lip }}$ where $\|\nabla F\|_{\text {Lip }}$ denotes the smallest Lipschitz constant of $\nabla F$. Now, for any $k \geq 1$, let us consider the following equation:

$$
\left\{\begin{array}{l}
\mathrm{d} X_{t}^{k}=A X_{t}^{k} \mathrm{~d} t+F_{k}\left(X_{t}^{k}\right) \mathrm{d} t+\mathrm{d} L_{t}+\mathrm{d} Z_{t}, \\
X_{0}^{k}=x
\end{array}\right.
$$

Let $\left\{X_{t}^{k, x}\right\}_{t \leq T}$ be its solution. Then it is easy to prove that

$$
\lim _{k \rightarrow \infty}\left|X_{t}^{k, x}-X_{t}\right|=0, \quad \text { and } \quad \lim _{k \rightarrow \infty} \int_{0}^{t}\left|X_{s}^{k, x}-X_{s}\right| \mathrm{d} s=0, \quad \forall t \geq 0 .
$$

Let $\left\{J_{t}^{k} \xi\right\}_{t \geq 0}$ be the derivative flow w.r.t. the initial value along $\xi$. Then

$$
J_{t}^{k} \xi=S(t) \xi+\int_{0}^{t} S(t-s) \nabla F^{k}\left(X_{s}^{k, x}\right) J_{s}^{k} \xi \mathrm{d} s .
$$

Furthermore,

$$
\sup _{k \geq 1}\left|J_{t}^{k} \xi\right| \leq \exp \left\{-\left(\gamma_{1}-\|\nabla F\|_{\infty}\right) t\right\}|\xi| .
$$

Now, by (52), (60) the triangle inequality we have

$$
\begin{aligned}
\left|J_{t}^{k} \xi-J_{t} \xi\right| \leq & \int_{0}^{t} e^{-\gamma_{1}(t-s)}\left|\nabla F_{k}\left(X_{s}^{k, x}\right) J_{s}^{k} \xi-\nabla F\left(X_{s}^{x}\right) J_{s} \xi\right| \mathrm{d} s \\
\leq & \int_{0}^{t} e^{-\gamma_{1}(t-s)}\left|\nabla F_{k}\left(X_{s}^{k, x}\right) J_{s}^{k} \xi-\nabla F_{k}\left(X_{s}^{k, x}\right) J_{s} \xi\right| \mathrm{d} s \\
& +\int_{0}^{t} e^{-\gamma_{1}(t-s)}\left|\nabla F_{k}\left(X_{s}^{k, x}\right) J_{s} \xi-\nabla F_{k}\left(X_{s}^{x}\right) J_{s} \xi\right| \mathrm{d} s \\
& +\int_{0}^{t} e^{-\gamma_{1}(t-s)}\left|\nabla F_{k}\left(X_{s}^{x}\right) J_{s} \xi-\nabla F\left(X_{s}^{x}\right) J_{s} \xi\right| \mathrm{d} s \\
\leq & \|\nabla F\|_{\infty} \int_{0}^{t} e^{-\gamma_{1}(t-s)}\left|J_{s}^{k} \xi-J_{s} \xi\right| \mathrm{d} s \\
& +\sup _{k \geq 1}\left\|\nabla^{2} F_{k}\right\|_{\infty} \int_{0}^{t} e^{\kappa(t-s)}\left|X_{s}^{k, x}-X_{s}^{x}\right| \exp \left\{\|\nabla F\|_{\infty} s\right\}|\xi| \mathrm{d} s \\
& +C_{A}^{2} \int_{0}^{t}\left\|\nabla F_{k}\left(X_{s}^{x}\right)-\nabla F\left(X_{s}^{x}\right)\right\| \exp \left\{\|\nabla F\|_{\infty} s\right\}|\xi| \mathrm{d} s .
\end{aligned}
$$


Using Gronwall's inequality and (59), one arrive at

(62) $\lim _{k \rightarrow \infty}\left|J_{t}^{k} \xi-J_{t} \xi\right|$

$$
\leq C \lim _{k \rightarrow \infty}\left\{\int_{0}^{t}\left|X_{s}^{k, x}-X_{s}^{x}\right| \mathrm{d} s+\int_{0}^{t}\left\|\nabla F_{k}\left(X_{s}^{x}\right)-\nabla F\left(X_{s}^{x}\right)\right\| \mathrm{d} s\right\}=0,
$$

where $C$ is a constant depending on $A, F$ and $t$. In view of (53), (59) and (62), one has

$$
\begin{aligned}
\lim _{k \rightarrow \infty} & \int_{0}^{t}\left|Q^{-1} J_{s}^{k} \xi-Q^{-1} J_{s} \xi\right| \mathrm{d} s \\
= & \lim _{k \rightarrow \infty} \int_{0}^{t} \mid \int_{0}^{s} Q^{-1} S(s-r) \nabla F\left(X_{r}^{k, x}\right) J_{r}^{k} \xi \mathrm{d} r \\
& \quad-\int_{0}^{s} Q^{-1} S(s-r) \nabla F\left(X_{r}^{x}\right) J_{r} \xi \mathrm{d} r \mid \mathrm{d} s \\
\leq & t \lim _{k \rightarrow \infty} \int_{0}^{t}\left\|Q^{-1} S(t-s)\right\|\left|\nabla F\left(X_{s}^{k, x}\right) J_{s}^{k} \xi-\nabla F\left(X_{s}^{x}\right) J_{s} \xi\right| \mathrm{d} s \\
= & t \int_{0}^{t}\left\|Q^{-1} S(t-s)\right\| \lim _{k \rightarrow \infty}\left|\nabla F\left(X_{s}^{k, x}\right) J_{s}^{k} \xi-\nabla F\left(X_{s}^{x}\right) J_{s} \xi\right| \mathrm{d} s=0,
\end{aligned}
$$

where in the second equality we use the conditions $\int_{0}^{t}\left\|Q^{-1} S(s)\right\| \mathrm{d} s<\infty$ and $F \in C_{b}^{1}(\mathbb{H}, \mathbb{H})$.

Now let us define

$$
P_{t}^{k, 1} f(x):=\mathbb{E}\left\{f\left(X_{t}^{k, x}\right) I_{\left[N_{t} \geq 1\right]}\right\}, \quad \forall f \in C_{b}^{2}(\mathbb{H}) .
$$

By Step 1, we have

$$
\begin{aligned}
\nabla_{\xi} & P_{t}^{k, 1} f(x) \\
= & -\mathbb{E}\left\{f\left(X_{t}^{k, x}\right) \frac{I_{\left[N_{t} \geq 1\right]}}{N_{t}}\right. \\
& \left.\times \int_{0}^{t} \int_{\mathbb{H}}\left(\left\langle z, Q^{-1} J_{s}^{k} \xi\right\rangle+\left\langle\nabla \log \rho(z), J_{s}^{k} \xi\right\rangle\right) \tilde{N}(\mathrm{~d} z, \mathrm{~d} s)\right\} .
\end{aligned}
$$

Observe that

$$
\lim _{k \rightarrow \infty}\left|\nabla_{\xi} P_{t}^{k, 1} f(x)-\nabla_{\xi} P_{t}^{1} f(x)\right| \leq\|\nabla f\|_{\infty} \lim _{k \rightarrow \infty} \mathbb{E}\left|J_{t}^{k} \xi-J_{t} \xi\right|=0,
$$

and

$$
\begin{aligned}
& \lim _{k \rightarrow \infty} \mathbb{E}\left|\int_{0}^{t} \int_{\mathbb{H}}\left(\left\langle z, Q^{-1} J_{s}^{k} \xi-Q^{-1} J_{s} \xi\right\rangle+\left\langle\nabla \log \rho(z),\left(J_{s}^{k} \xi-J_{s} \xi\right)\right\rangle\right) \tilde{N}(\mathrm{~d} z, \mathrm{~d} s)\right| \\
& \leq C \lim _{k \rightarrow \infty}\left\{\int_{0}^{t}\left|Q^{-1} J_{s}^{k} \xi-Q^{-1} J_{s} \xi\right| \mathrm{d} s+\int_{0}^{t}\left|J_{s}^{k} \xi-J_{s} \xi\right| \mathrm{d} s\right\}=0 .
\end{aligned}
$$

We finish the proof by letting $k \rightarrow \infty$ in (64). 
3.2. Proof of Theorem 2. We divide the proof into two steps.

Step 1: Assume $F \in C_{b}^{2}(\mathbb{H}, \mathbb{H})$. First, we recall that

$$
\left|J_{t} \xi\right| \leq \exp \left\{-\left(\gamma_{1}-\|\nabla F\|_{\infty}\right) t\right\}|\xi| .
$$

By direct computation,

$$
\mathbb{E}\left\{\frac{I_{\left[N_{t} \geq 1\right]}}{\left(N_{t}\right)^{2}}\right\}=\sum_{n=1}^{\infty} \frac{e^{-\lambda t}(\lambda t)^{n}}{n^{2} n !} \leq \frac{6 e^{-\lambda t}}{(\lambda t)^{2}} \sum_{n=1}^{\infty} \frac{(\lambda t)^{n+2}}{(n+2) !} \leq \frac{6}{(\lambda t)^{2}} .
$$

By Hölder inequality, we have

$$
\begin{array}{rl}
\int_{0}^{t} & \mathbb{E}\left|Q^{-1} J_{s} \xi\right|^{2} \mathrm{~d} s \\
= & \int_{0}^{t} \mathbb{E}\left|Q^{-1} S(s) \xi+\int_{0}^{s} Q^{-1} S(s-r) \nabla F\left(X_{r}\right) J_{r} \xi \mathrm{d} r\right|^{2} \mathrm{~d} s \\
\leq & 2 \int_{0}^{t}\left|Q^{-1} S(s) \xi\right|^{2} \mathrm{~d} s+2 \int_{0}^{t} \mathbb{E}\left|\int_{0}^{s} Q^{-1} S(s-r) \nabla F\left(X_{r}\right) J_{r} \xi \mathrm{d} r\right|^{2} \mathrm{~d} s \\
\leq & 2\|\nabla F\|_{\infty}^{2}|\xi|^{2} \\
& \times \int_{0}^{t}\left\{s \int_{0}^{s}\left\|Q^{-1} S(s-r)\right\|^{2} \exp \left\{-2\left(\gamma_{1}-\|\nabla F\|_{\infty}\right) r\right\} \mathrm{d} r\right\} \mathrm{d} s \\
& +2|\xi|^{2} \int_{0}^{t}\left\|Q^{-1} S(s)\right\|^{2} \mathrm{~d} s \\
= & 2\|\nabla F\|_{\infty}^{2}|\xi|^{2} \Gamma_{t}+2|\xi|^{2} \int_{0}^{t}\left\|Q^{-1} S(s)\right\|^{2} \mathrm{~d} s,
\end{array}
$$

where

$$
\Gamma_{t}:=\int_{0}^{t}\left\{s \int_{0}^{s}\left\|Q^{-1} S(s-r)\right\|^{2} \exp \left\{-2\left(\gamma_{1}-\|\nabla F\|_{\infty}\right) r\right\} \mathrm{d} r\right\} \mathrm{d} s .
$$

Since $\lim _{t \rightarrow \infty} \frac{\int_{0}^{t}\left\|Q^{-1} S(s)\right\|^{2} \mathrm{~d} s}{t}<\infty$, then $\lim _{t \rightarrow \infty}\left\|Q^{-1} S(t)\right\|^{2}<\infty$. Moreover,

$$
\begin{aligned}
\lim _{t \rightarrow \infty} \frac{\Gamma_{t}}{t^{2}} & =\lim _{t \rightarrow \infty} \frac{\int_{0}^{t}\left\{s \int_{0}^{s}\left\|Q^{-1} S(s-r)\right\|^{2} \exp \left\{-2\left(\gamma_{1}-\|\nabla F\|_{\infty}\right) r\right\} \mathrm{d} r\right\} \mathrm{d} s}{t^{2}} \\
& \leq \frac{1}{2} \lim _{t \rightarrow \infty} \int_{0}^{t}\left\|Q^{-1} S(t-s)\right\|^{2} \exp \left\{-2\left(\gamma_{1}-\|\nabla F\|_{\infty}\right) s\right\} \mathrm{d} s \\
& =\lim _{t \rightarrow \infty} \frac{\int_{0}^{t}\left\|Q^{-1} S(s)\right\|^{2} \exp \left\{2\left(\gamma_{1}-\|\nabla F\|_{\infty}\right) s\right\} \mathrm{d} s}{2 \exp \left\{2\left(\gamma_{1}-\|\nabla F\|_{\infty}\right) t\right\}} \\
& \leq \frac{\lim _{t \rightarrow \infty}\left\|Q^{-1} S(t)\right\|^{2}}{4\left(\gamma_{1}-\|\nabla F\|_{\infty}\right)}<\infty .
\end{aligned}
$$


Therefore, there exists $C_{1}>0$ independent of $t$ and $F$, such that

$$
\left(\sup _{t \geq 1} \frac{\Gamma_{t}}{t^{2}}\right)+\left(\sup _{t \geq 1} \frac{\int_{0}^{t}\left\|Q^{-1} S(s)\right\|^{2} \mathrm{~d} s}{t^{2}}\right) \leq \frac{C_{1}}{\gamma_{1}-\|\nabla F\|_{\infty}} .
$$

Recalling that $\nu$ is finite measure and by Theorem 1, (65)-(68) one has for each $t \geq 1$

$$
\begin{aligned}
\mid \nabla_{\xi} & P_{t}^{1} f(x) \mid \\
= & \left|-\mathbb{E}\left\{f\left(X_{t}^{x}\right) \frac{I_{\left[N_{t} \geq 1\right]}}{N_{t}} \int_{0}^{t} \int_{\mathbb{H}}\left(\left\langle z, Q^{-1} J_{s} \xi\right\rangle+\left\langle\nabla \log \rho(z), J_{s} \xi\right\rangle\right) \widetilde{N}(\mathrm{~d} z, \mathrm{~d} s)\right\}\right| \\
\leq & \|f\|_{\infty}\left\{\left\{\int_{\mathbb{H}}|z|^{2} \nu(\mathrm{d} z) \mathbb{E} \frac{I_{\left[N_{t} \geq 1\right]}}{\left(N_{t}\right)^{2}} \int_{0}^{t} \mathbb{E}\left|Q^{-1} J_{s} \xi\right|^{2} \mathrm{~d} s\right\}^{\frac{1}{2}}\right. \\
& \left.+2\|\nabla \rho\|_{\infty} \int_{0}^{t} \mathbb{E}\left|J_{s} \xi\right| \mathrm{d} s\right\} \\
\leq & \|f\|_{\infty}\left\{\left\{\int_{\mathbb{H}}|z|^{2} \nu(\mathrm{d} z) \mathbb{E} \frac{I_{\left[N_{t} \geq 1\right]}}{\left(N_{t}\right)^{2}} \int_{0}^{t} \mathbb{E}\left|Q^{-1} J_{s} \xi\right|^{2} \mathrm{~d} s\right\}^{\frac{1}{2}}+\frac{2\|\nabla \rho\|_{\infty}|\xi|}{\gamma_{1}-\|\nabla F\|_{\infty}}\right\} \\
\leq & \|f\|_{\infty}|\xi|\left\{\left\{12 \int_{\mathbb{H}}|z|^{2} \nu(\mathrm{d} z) \frac{\|\nabla F\|_{\infty}^{2} \Gamma_{t}+\int_{0}^{t}\left\|Q^{-1} S(s)\right\|^{2} \mathrm{~d} s}{\lambda^{2} t^{2}}\right\}^{\frac{1}{2}}\right. \\
& +\frac{2\|\nabla \rho\|_{\infty}}{\left.\gamma_{1}-\|\nabla F\|_{\infty}\right\}} \\
\leq & \|f\|_{\infty}|\xi| \frac{\left\{12 C_{1} \int_{\mathbb{H}}|z|^{2} \nu(\mathrm{d} z)\left(\|\nabla F\|_{\infty}^{2}+1\right)\right\}^{\frac{1}{2}}+2\|\nabla \rho\|_{\infty}}{(\lambda \wedge 1)\left(\left(\gamma_{1}-\|\nabla F\|_{\infty}\right) \wedge 1\right)} .
\end{aligned}
$$

Therefore, for each $x, y \in \mathbb{H}$ and $t \geq 1$

$$
\begin{aligned}
\left|P_{t} f(x)-P_{t} f(y)\right| & \\
= & \left|P_{t}^{1} f(x)-P_{t}^{1} f(y)\right|+\left|\mathbb{E}\left\{f\left(X_{t}^{x}\right) I_{\left[N_{t}=0\right]}\right\}-\mathbb{E}\left\{f\left(X_{t}^{y}\right) I_{\left[N_{t}=0\right]}\right\}\right| \\
\leq & \|f\|_{\infty}|x-y| \frac{\left\{12 C_{1} \int_{\mathbb{H}}|z|^{2} \nu(\mathrm{d} z)\left(\|\nabla F\|_{\infty}^{2}+1\right)\right\}^{\frac{1}{2}}+2\|\nabla \rho\|_{\infty}}{(\lambda \wedge 1)\left(\left(\gamma_{1}-\|\nabla F\|_{\infty}\right) \wedge 1\right)} \\
& +2\|f\|_{\infty} e^{-\lambda t} .
\end{aligned}
$$

Step 2: Assume $F$ is Lipschitz continuous. Then there exist $\left\{F_{n}\right\}_{n \geq 1} \subset$ $C_{b}^{2}(\mathbb{H}, \mathbb{H})$ such that $F_{n} \rightarrow F$ as $n \rightarrow \infty$ in pointwise sense and $\sup _{n \geq 1}\left\|\nabla F_{n}\right\|_{\infty} \leq\|F\|_{\text {Lip }}$. Now let us consider the following equation:

$$
\left\{\begin{array}{l}
\mathrm{d} Y_{t}^{n}=A Y_{t}^{n} \mathrm{~d} t+F_{n}\left(Y_{t}^{n}\right) \mathrm{d} t+\mathrm{d} L_{t}+\mathrm{d} Z_{t}, \\
Y_{0}^{n}=x
\end{array}\right.
$$


Let $\left\{P_{n, t}\right\}_{t \geq 0}$ be the transition semigroup of the solution to Eq. (70). Then it is easy to prove that

$$
\lim _{n \rightarrow \infty} P_{n, t} f(y)=P_{t} f(y), \quad \forall y \in \mathbb{H}, \forall f \in C_{b}^{2}(\mathbb{H}) .
$$

Now it follows from (69) that for any $t \geq 1$ and $f \in C_{b}^{2}(\mathbb{H})$

$$
\begin{array}{rl}
\mid P_{n, t} & f(x)-P_{n, t} f(y) \mid \\
\leq & \|f\|_{\infty}|x-y| \frac{\left\{12 C_{1} \int_{\mathbb{H}}|z|^{2} \nu(\mathrm{d} z)\left(\left\|\nabla F_{n}\right\|_{\infty}^{2}+1\right)\right\}^{\frac{1}{2}}+2\|\nabla \rho\|_{\infty}}{(\lambda \wedge 1)\left(\gamma_{1}-\left\|\nabla F_{n}\right\|_{\infty}\right)} \\
& +2\|f\|_{\infty} e^{-\lambda t} \\
\leq & \|f\|_{\infty}|x-y| \frac{\left\{12 C_{1} \int_{\mathbb{H}}|z|^{2} \nu(\mathrm{d} z)\left(\|F\|_{\text {Lip }}^{2}+1\right)\right\}^{\frac{1}{2}}+2\|\nabla \rho\|_{\infty}}{(\lambda \wedge 1)\left(\left(\gamma_{1}-\|F\|_{\text {Lip }}\right) \wedge 1\right)} \\
& +2\|f\|_{\infty} e^{-\lambda t} .
\end{array}
$$

Letting $n \rightarrow \infty$, we get

$$
\left|P_{t} f(x)-P_{t} f(y)\right| \leq C\|f\|_{\infty}\left\{|x-y|+e^{-\lambda t}\right\}, \quad \forall t \geq 1,
$$

for some constant $C>0$ independent of $t$. Observe that for each $x, y \in \mathbb{H}$,

$$
\begin{aligned}
\mathbb{E}\left|X_{t}^{x}-X_{t}^{y}\right| & \leq|S(t)(x-y)|+\mathbb{E} \int_{0}^{t}\left|S(t-s)\left(F\left(X_{s}^{x}\right)-F\left(X_{s}^{y}\right)\right)\right| \mathrm{d} s \\
& \leq e^{-\gamma_{1} t}|x-y|+\|F\|_{\text {Lip }} \int_{0}^{t} e^{-\gamma_{1}(t-s)} \mathbb{E}\left|X_{s}^{x}-X_{s}^{y}\right| \mathrm{d} s .
\end{aligned}
$$

Then Gronwall's inequality yields

$$
\mathbb{E}\left|X_{t}^{x}-X_{t}^{y}\right| \leq \exp \left\{\left(-\gamma_{1}+\|F\|_{\text {Lip }}\right) t\right\}|x-y|, \quad \forall x, y \in \mathbb{H} .
$$

Combining this with (71) and using the Markov property, we have for $t>s$

$$
\begin{aligned}
& \left|P_{t} f(x)-P_{t} f(y)\right| \\
& \quad \leq \mathbb{E}\left|P_{s} f\left(X_{t-s}^{x}\right)-P_{s} f\left(X_{t-s}^{y}\right)\right| \\
& \quad \leq 2 C\|f\|_{\infty}\left\{\mathbb{E}\left|X_{t-s}^{x}-X_{t-s}^{y}\right|+e^{-\lambda s}\right\} \\
& \quad \leq 2 C\|f\|_{\infty}\left\{\exp \left\{-\left(\gamma_{1}-\|F\|_{\text {Lip }}\right)(t-s)\right\}|x-y|+e^{-\lambda s}\right\} .
\end{aligned}
$$

Let $t>\frac{\left(\gamma_{1}-\|F\|_{\text {Lip }}+\lambda\right)}{\gamma_{1}-\|F\|_{\text {Lip }}}$ and take $s=\frac{\left(\gamma_{1}-\|F\|_{\text {Lip }}\right) t}{\gamma_{1}-\|F\|_{\text {Lip }}+\lambda}$ in $(72)$, then

$$
\begin{aligned}
& \left|P_{t} f(x)-P_{t} f(y)\right| \\
& \quad \leq C\|f\|_{\infty}(1+|x-y|) \exp \left\{-\frac{\lambda\left(\gamma_{1}-\|F\|_{\text {Lip }}\right)}{\lambda+\gamma_{1}-\|F\|_{\text {Lip }}} t\right\} .
\end{aligned}
$$


Therefore,

$$
\begin{aligned}
\left\|P_{t}(x, \cdot)-P_{t}(y, \cdot)\right\|_{\text {Var }} & :=\frac{1}{2} \sup _{f \in \mathbb{B}_{b}(\mathbb{H}),\|f\|_{\infty} \leq 1}\left|P_{t} f(x)-P_{f}(y)\right| \\
& =\frac{1}{2} \sup _{f \in C_{b}^{2}(\mathbb{H}),\|f\|_{\infty} \leq 1}\left|P_{t} f(x)-P_{f}(y)\right| \\
& \leq C(1+|x-y|) \exp \left\{-\frac{\lambda\left(\gamma_{1}-\|F\|_{\text {Lip }}\right)}{\lambda+\gamma_{1}-\|F\|_{\text {Lip }}} t\right\} .
\end{aligned}
$$

The proof is completed by noting that the inequality trivially holds with a suitable constant $C>0$ for $t \leq \frac{\left(\gamma_{1}-\|F\|_{\text {Lip }}+\lambda\right)}{\left(\gamma_{1}-\|F\|_{\text {Lip }}\right)}$. The proof is finished.

Acknowledgment. The authors are grateful to the referee's valuable suggestions.

\section{REFERENCES}

[1] S. Albeverio, V. Mandrekar and B. Rüdiger, Existence of mild solutions for stochastic differential equations and semilinear equations with non-Gaussian Lévy noise, Stochastic Process. Appl. 119 (2009), 835-863. MR 2499860

[2] S. Albeverio, B. Rüdiger and J. L. Wu, Invariant measures and symmetry property of Lévy type operators, Potential Anal. 13 (2000), 147-168. MR 1782254

[3] V. Bally, M. P. Bavouzet and M. Messaoud, Integration by parts formula for locally smooth laws and applications to sensitivity computations, Ann. Appl. Probab. 17 (2007), 33-66. MR 2292579

[4] R. F. Bass and M. Cranston, The Malliavin calculus for pure jump processes and applications to local time, Ann. Probab. 14 (1986), 490-532. MR 0832021

[5] K. Bichteler, J. B. Gravereaux and J. Jacod, Malliavin calculus for processes with jumps, Gordon \& Breach, New York, 1987. MR 1008471

[6] J. M. Bismut, Calcul des variations stochastique et processus de saut, Z. Wahrscheinlichkeiststheor. Verw. Geb. 63 (1983), 147-235. MR 0701527

[7] J. M. Bismut, Large deviations and the Malliavin calculus, Birkhäuser, Boston, 1984. MR 0755001

[8] B. Bottcher, R. L. Schilling and J. Wang, Constructions of coupling processes for Lévy processes, Stochastic Process. Appl. 121 (2011), 1201-1216. MR 2794973

[9] G. Da Prato and J. Zabczyk, Stochastic equations in infinite dimensions, Cambridge University Press, Cambridge, 1992. MR 1207136

[10] G. Da Prato and J. Zabczyk, Ergodicity for infnite diemsnional systems, Cambridge University Press, Cambridge, 1996. MR 1417491

[11] G. Di Nunno, B. Øsendal and F. Proske, Malliavin calculus for Lévy processes with applications to finance, Springer, Berlin, 2009. MR 2460554

[12] Z. Dong, L. H. Xu and X. C. Zhang, Exponential ergodicity of stochastic Burgers equations driven by $\alpha$-stable processes, J. Stat. Phys. 154 (2014), 929-949. MR 3164597

[13] K. D. Elworthy and X. M. Li, Formulae for the derivatives of heat semigroups, J. Funct. Anal. 125 (1994), 252-286. MR 1297021

[14] E. Hausenblas, Existence, uniqueness and regularity of parabolic SPDEs driven by Poisson random measure, Electron. J. Probab. 10 (2005), 1496-1546. MR 2191637

[15] A. M. Kulik, Exponential ergodicity of the solutions to SDEs with a jump noise, Stochastic Process. Appl. 119 (2009), 602-632. MR 2494006 
[16] C. Marinelli, C. Prévôt and M. Röckner, Regular dependence on initial data for stochastic evolution equations with multiplicative Poisson noise, J. Funct. Anal. 258 (2010), 616-649. MR 2557949

[17] C. Mueller, The heat equation with Lévy noise, Stochastic Process. Appl. 74 (1998), 67-82. MR 1624088

[18] C. Mueller, L. Mytnik and A. Stan, The heat equation with time-independent multiplicative stable Lévy noise, Stochastic Process. Appl. 116 (2006), 70-100. MR 2186840

[19] L. Mytnik, Stochastic partial differential equation driven by stable noise, Probab. Theory Related Fields 123 (2002), 157-201. MR 1900321

[20] J. R. Norris, Integration by parts for jump processes, Seminaire de probabilites XXII, Lect. Notes. Math., vol. 1321, 1988, pp. 271-315. MR 0960534

[21] S. Peszat and J. Zabczyk, Stochastic partial differential equations with Lévy noise: Evlotion equations approach, Cambridge University Press, Cambridge, 2007. MR 2356959

[22] E. Priola, A. Shirikyan, L. H. Xu and J. Zabczyk, Exponential ergodicity and regularity for equations with Lévy noise, Stochastic Process. Appl. 122 (2012), 106-133. MR 2860444

[23] E. Priola, L. H. Xu and J. Zabczyk, Exponential mixing for some SPDEs with Lévy noise, Stoch. Dyn. 11 (2011), 521-534. MR 2836539

[24] E. Priola and J. Zabczyk, Structural properties of semilinear SPDEs driven by cylindrical stable processes, Probab. Theory Related Fields 149 (2011), 97-137. MR 2773026

[25] M. Röckner and T. S. Zhang, Stochastic evolution equations of jump type: Existence, uniqueness and large deviation principles, Potential Anal. 26 (2007), 255-279. MR 2286037

[26] R. L. Schilling and J. Wang, On the coupling property of Lévy processes, Ann. Inst. Henri Poincaré Probab. Stat. 47 (2011), 1147-1159. MR 2884228

[27] R. L. Schilling and J. Wang, On the coupling property and the Liouville theorem for Ornstein-Uhlenbeck processes, J. Evol. Equ. 12 (2012), 119-140. MR 2891204

[28] Y. L. Song, Derivative formula and exponential convergence for semilinear SPDEs driven by Lévy processes, Statist. Probab. Lett. 89 (2014), 99-109. MR 3191467

[29] Y. L. Song and X. C. Zhang, Regularity of density for SDEs driven by degenerate Lévy noises, Electron. J. Probab. 20 (2015), 1-27. MR 3325091

[30] A. Takeuchi, Bismut-Elworthy-Li-type formula for stochastic differential equations with jumps, J. Theoret. Probab. 23 (2010), 576-604. MR 2644877

[31] F. Y. Wang, Gradient estimate for Ornstein-Uhlenbeck jump processes, Stochastic Process. Appl. 121 (2011), 466-478. MR 2763092

[32] F. Y. Wang, Harnack inequalities for stochastic partial differential equations, Springer, New York, 2013. MR 3099948

[33] F. Y. Wang, Derivative formula and Harnack inequality for jump processes, Stoch. Anal. Appl. 32 (2014), 30-49. MR 3175813

[34] F. Y. Wang and J. Wang, Coupling and strong Feller for jump processes on Banach spaces, Stochastic Process. Appl. 123 (2013), 1588-1615. MR 3027892

[35] X. C. Zhang, Derivative formula and gradient estimate for SDEs driven by $\alpha$-stable processes, Stochastic Process. Appl. 123 (2013), 1213-1228. MR 3016221

Yulin Song, Department of Mathematics, Nanjing University, Nanjing, Jiangsu

Province, 210093, P.R. China

E-mail address: songyl@amss.ac.cn

Tiange Xu, School of Mathematics, University of CAS, Beijing, 100049, P.R. CHINA

E-mail address: xutiange@gmail.com 